\title{
Origin of the abundance patterns in Galactic globular clusters: constraints on dynamical and chemical properties of globular clusters
}

\author{
T. Decressin ${ }^{1}$, C. Charbonnel ${ }^{1,2}$, and G. Meynet ${ }^{1}$ \\ 1 Observatoire de Genève, Université de Genève, 51 ch. des Maillettes, 1290 Sauverny, Switzerland \\ e-mail: Thibaut.Decressin@obs.unige.ch \\ 2 Laboratoire d'Astrophysique de Toulouse et Tarbes, CNRS UMR 5572, OMP, Université Paul Sabatier Toulouse 3, 14 Av. E. Belin, \\ 31400 Toulouse, France
}

Received 6 August 2007 / Accepted 18 September 2007

ABSTRACT

\begin{abstract}
Aims. We analyse the effects of a first generation of fast rotating massive stars on the dynamical and chemical properties of globular clusters.

Methods. We use stellar models of fast rotating massive stars, losing mass through a slow mechanical equatorial winds to produce material rich in $\mathrm{H}$-burning products. We propose that stars with high $\mathrm{Na}$ and low $\mathrm{O}$ abundances (hereafter anomalous stars) are formed from matter made of slow winds of individual massive stars and of interstellar matter. The proportion of slow wind and of interstellar material is fixed in order to reproduce the observed Li-Na anticorrelation in NGC 6752.

Results. In the case that globular clusters, during their lifetime, did not lose any stars, we found that to reproduce the observed ratio of normal to anomalous stars, a flat initial mass function (IMF) is needed, with typically a slope $x=0.55$ (a Salpeter's IMF has $x=1.35$ ). In the case that globular clusters suffer from an evaporation of normal stars, the IMF slope can be steeper: to have $x=1.35$, about $96 \%$ of the normal stars would be lost. We make predictions for the distribution of stars as a function of their $[\mathrm{O} / \mathrm{Na}]$ and obtain quite reasonable agreement with that one observed for NGC 6752. Predictions for the number fraction of stars with different values of helium, of the ${ }^{12} \mathrm{C} /{ }^{13} \mathrm{C}$ and ${ }^{16} \mathrm{O} /{ }^{17} \mathrm{O}$ ratios are discussed, as well as the expected relations between values of [O/Na] and those of helium, of $[\mathrm{C} / \mathrm{N}]$, of ${ }^{12} \mathrm{C} /{ }^{13} \mathrm{C}$ and of ${ }^{16} \mathrm{O} /{ }^{17} \mathrm{O}$. Future observations might test these predictions. We also provide predictions for the present day mass of the clusters expressed in units of mass of the gas used to form stars, and for the way the present day mass is distributed between the first and second generation of stars and the stellar remnants.
\end{abstract}

Key words. globular clusters: general - globular clusters: individual: NGC 6752 - stars: abundances stars: luminosity function, mass function - stars: mass-loss - stars: rotation

\section{Introduction}

It has long been known that globular cluster stars present some striking anomalies in their content in light elements ${ }^{1}$ : while in all the Galactic globular clusters studied so far one finds "normal" stars with detailed chemical composition similar to those of field stars of same metallicity (i.e., same $[\mathrm{Fe} / \mathrm{H}]$ ), one also observes numerous "anomalous" main sequence and red giant stars that are simultaneously deficient (to various degrees) in C, $\mathrm{O}$, and $\mathrm{Mg}$, and enriched in $\mathrm{N}, \mathrm{Na}$, and $\mathrm{Al}$ (for recent reviews see Gratton et al. 2004; Charbonnel 2005). Additionally, the abundance of the fragile $\mathrm{Li}$ was found to be anticorrelated with that of $\mathrm{Na}$ in turnoff stars in a couple of globular clusters (Pasquini et al. 2005; Bonifacio et al. 2007).

It is clear now that these chemical peculiarities were inherited at birth by the low-mass stars we observe today in globular clusters, and that their root cause is H-burning through the CNOcycle and the NeNa- and MgAl-chains that occurred in an early generation of more massive and faster evolving globular cluster stars $^{2}$ (see Prantzos \& Charbonnel 2006, hereafter PC06, and

1 On the contrary, the content in heavy elements (i.e., Fe-group, $\alpha$-elements) is fairly constant from star to star in any well-studied individual Galactic globular cluster (with the notable exception of $\omega$ Cen).

${ }^{2}$ As in field stars, the surface abundances of $\mathrm{Li}, \mathrm{C}$, and $\mathrm{N}$ also vary in globular cluster stars due to in situ evolutionary processes (i.e., first references therein). In other words, compelling evidence leads us to believe that at least two generations of stars succeeded in all Galactic globular clusters during their infancy. The first one corresponds to the bulk of "normal" stars born with the pristine composition of the protocluster gas; these objects are those with the highest $\mathrm{O}$ and $\mathrm{Mg}$ and the lowest $\mathrm{Na}$ and $\mathrm{Al}$ abundances also found in their field contemporaries. The second generation contains the stars born out of material polluted to various degrees by the ejecta of more massive stars, and which present lower $\mathrm{O}$ and $\mathrm{Mg}$ and higher $\mathrm{Na}$ and $\mathrm{Al}$ abundances than their first generation counterparts.

Any model attempting to explain the chemical properties of globular cluster stars should give an answer to the following questions: (1) Which type of stars produced the material enriched in H-burning products? (2) What is the physical mechanism responsible for selecting only material bearing the signatures of H-processing? (3) Why does this process occur only in globular clusters? $?^{3}$

dredge-up and thermohaline instability on the red giant branch; see Charbonnel \& Zahn 2007, and references therein). We will not address this point in the present paper.

${ }^{3}$ Indeed up to now the peculiar chemical patterns observed in globular clusters, i.e., the $\mathrm{O}-\mathrm{Na}$ and $\mathrm{Mg}$ - $\mathrm{Al}$ anticorrelations, have not been found in field stars. 


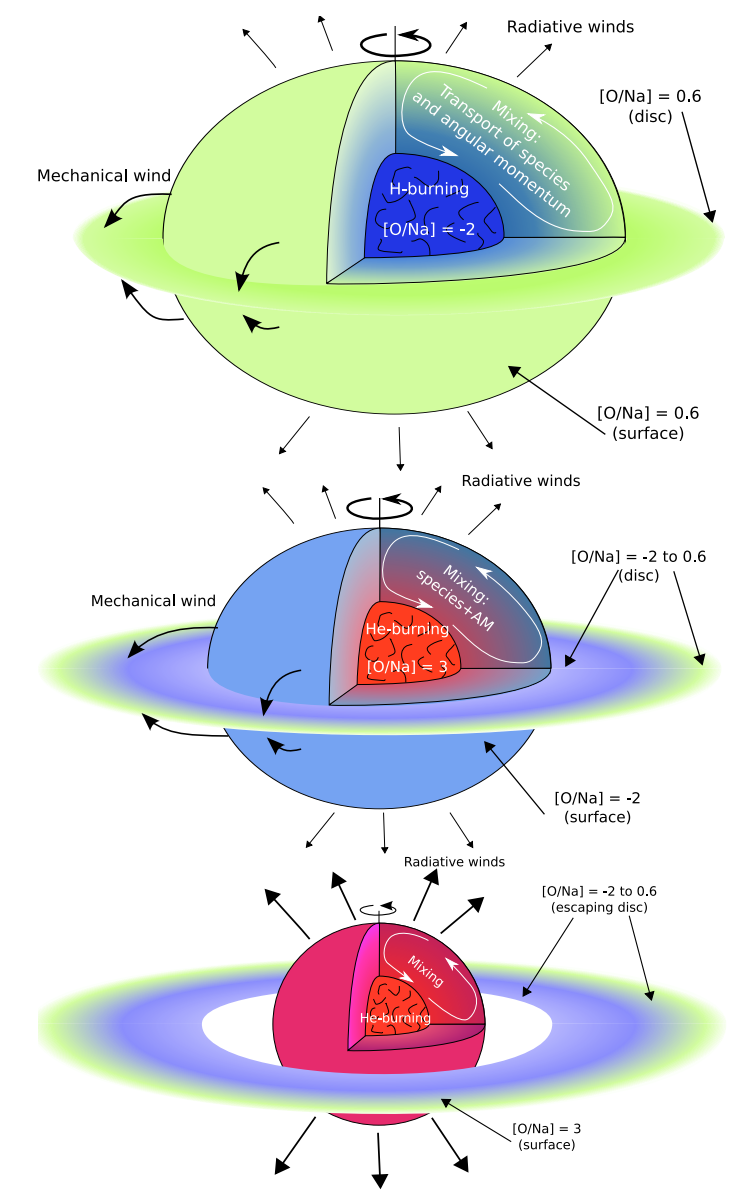

Fig. 1. Schematic view of the evolution of fast rotating massive stars. The colours reflect the chemical composition of the various stellar regions and of the disc (see text for details). (Top) During the main sequence, a slow outflowing equatorial disc forms and dominates matter ejection with respect to radiative winds. (Middle) At the beginning of central He-burning, the composition of the disc material spans the range in $[\mathrm{O} / \mathrm{Na}]$ observed today in low-mass cluster stars. The star has already lost an important fraction of its initial mass. (Bottom) Due to heavy mass loss, the star moves away from critical velocity and does not supply its disc anymore; radiatively-driven fast wind takes over before the products of He-burning reach the stellar surface.

On the basis of considerations on IMF of the potential "polluting stars", PC06 proposed massive stars (i.e., stars with an initial mass higher than $\sim 10 M_{\odot}$ ) as possible "culprits". In Decressin et al. (2007, hereafter Paper I) we extended this idea and developed stellar models sustaining our so-called "wind of fast rotating massive stars" (hereafter WFRMS) scenario that gives satisfactory answers to the previous questions, and that we recall below.

\section{The wind of fast rotating stars scenario-main guidelines}

In our WFRMS scenario, a first generation of stars forms from proto-cluster gas already pre-enriched in heavy metals during the halo chemical evolution (see PC06 for a more extended discussion). They have thus the same initial composition as their field contemporaries; they present in particular the highest $[\mathrm{O} / \mathrm{Na}] \mathrm{ra}-$ tio we can observe today in any individual globular cluster. We assume that stars with masses between 0.1 and $120 M_{\odot}$ form at that stage, with an initial mass function (IMF) that has to be determined.

Based on observations supporting the view that stars born in dense environments have faster rotational velocities than stars born in loose aggregates (Keller 2004; Strom et al. 2005; Huang $\&$ Gies 2006; Dufton et al. 2006), we further assume that the first generation massive stars are fast rotators. The evolution of such an object is shown in Fig. 1. The colours reflect the chemical composition of the various stellar regions: Green corresponds to the initial chemical composition, blue and red are respectively for material loaded in $\mathrm{H}$ - and He-burning products. The typical $[\mathrm{O} / \mathrm{Na}]$ value in the various stellar regions is indicated. In the protostars of first generation, $[\mathrm{O} / \mathrm{Na}]$ is typically $\sim 0.6$ (see Sect. 3).

Fast rotating massive stars with typical time averaged velocity of $500 \mathrm{~km} \mathrm{~s}^{-1}$ on the main sequence easily reach the critical velocity ${ }^{4}$ at the beginning of their evolution and remain near the critical limit during the rest of the main sequence and part of the central He-burning phase (see Paper I and Ekstroem et al., submitted). As a consequence, during those phases they lose large amounts of material through a mechanical wind ${ }^{5}$, which probably leads to the formation of a slow outflowing Keplerian equatorial disc. This is the kind of process believed to occur in $\mathrm{Be}$ stars (Porter \& Rivinius 2003).

The material ejected in the disc has two interesting characteristics: (1) due to rotational mixing that transports the products of the CNO cycle and of the $\mathrm{Ne}-\mathrm{Na}$ and $\mathrm{Mg}-\mathrm{Al}$ chains from the core to the stellar surface, it bears the signatures of H-burning and presents abundance patterns similar to the chemical anomalies observed in the second generation stars as indicated by the range of $[\mathrm{O} / \mathrm{Na}]^{6} ;(2)$ it is released into the circumstellar environment with a very low velocity and thus can easily be retained in the shallow potential well of the globular cluster. In the following we shall call it the slow wind.

The disc-star configuration lasts throughout the main sequence for stars close to or at critical velocity; for the most massive stars that are at the $\Omega \Gamma$-limit (i.e., when the surface luminosity is near the Eddington limit), it persists in the LBV phase. When the star evolves away from the critical limit due to heavy mass loss, the radiatively-driven fast wind takes over and the disc is supplied by the star no longer. This happens during the central He-burning phase, before the He-burning products reach the stellar surface and contaminate the slow wind component (bottom panel of Fig. 1). From that moment on, the high-speed material ejected by the star and by the potential supernova will escape the globular cluster. We proposed that this filtering mechanism, which retains in the potential well of the globular cluster only the slow stellar ejecta, is the physical mechanism responsible for supplying the required $H$-processed material for forming the stars of the second generation.

In the vicinity of massive stars, star formation may occur, triggered for instance by the ionisation front. The large

\footnotetext{
4 The critical velocity is the velocity at the equator such that the centrifugal acceleration balances the gravity.

${ }^{5}$ For example, our $60 M_{\odot}$ model loses $24.3 M_{\odot}$ in its slow wind.

${ }^{6}$ Early on the main sequence, the $[\mathrm{O} / \mathrm{Na}]$ value in the slow wind is pristine (top panel). As mixing proceeds, matter with $[\mathrm{O} / \mathrm{Na}]$ typical of H-burning (i.e., - 2) is ejected in the slow wind (middle panel). In Paper I, we showed that the yields of fast rotating massive stars (with an initial mass higher than $\sim 20 M_{\odot}$ ) properly explain the O-Na anticorrelation observed today in Galactic globular clusters. We note however that in order to reproduce the lowest $\mathrm{Mg}$ values observed we had to postulate an increase of the ${ }^{24} \mathrm{Mg}(p, \gamma)^{25} \mathrm{Al}$ nuclear reaction rate with respect to the published values.
} 
star-to-star spread in light elements observed today indicates that all the material ejected by the first generation massive stars did not have the time to be fully mixed before being recycled in the second generation. There is however clear evidence from the observed behaviour of both $\mathrm{O}$ and $\mathrm{Li}$ that the material ejected in the slow wind is mixed with pristine interstellar matter (ISM; see Kudryashov \& Tutukov 1988; Denisenkov \& Denisenkova 1989, 1990; Langer et al. 1993; Langer \& Hoffman 1995; Prantzos et al. 2007). This suggests that star formation may have occurred in the vicinity of individual massive stars from clumps in the slow wind interacting with pristine gas. As discussed before, the material ejected in the slow wind presents various degrees of enrichment in H-burning products. At the beginning of the disc-star phase, pristine material is ejected giving birth to stars with a composition similar to that of the first generation. As time proceeds, the slow wind becomes more and more polluted in $\mathrm{H}$ burning products (see Fig. 1) and forms stars that are more and more "anomalous". This phase lasts only a few million years (e.g., 4.5 Myr for a $60 M_{\odot}$ star).

At this stage, the WFRMS scenario gives promising answers to the questions raised in Sect. 1, but it does not address the question of the dynamical evolution of the cluster as a whole. This is what we explore in the present paper, as well as other consequences regarding the He content and the carbon and oxygen isotopic ratios of the second generation stars. Based on quantitative theoretical yields of our models for fast rotating massive stars (Paper I), we first address the question of the IMF of the polluter stars. We use the method developed by PC06 which relies on the observed $[\mathrm{O} / \mathrm{Na}]$ distribution to derive the amount of polluted material with respect to the pristine composition. In Sect. 3 we present the general equations giving the number ratio of anomalous to normal stars, the number fraction of stars born with a specific chemical composition, as well as the ratio of the mass of stellar remnants to stars in the cluster today. The two scenarios that we have investigated regarding the early evolution of a Galactic globular cluster are presented in Sect. 4. Application to the case of NGC 6752 is discussed in Sect. 5. Section 6 presents our predictions for the helium content and the isotopic ratios ${ }^{12} \mathrm{C} /{ }^{13} \mathrm{C}$ and ${ }^{16} \mathrm{O} /{ }^{17} \mathrm{O}$ respectively. Discussion and conclusions are the subject of Sect. 7.

\section{General equations}

Before deriving the equations that will be necessary in this paper, we recall that only the stars with an initial mass lower or equal to $\sim 0.8 M_{\odot}$ are still alive today. This corresponds approximately to the turn-off mass of $\sim 12$ Gyr globular clusters. We will call them the long-lived (hereafter LL) stars. More massive stars has died previously and formed dark stellar remnants.

We note also that, according to the nucleosynthetic predictions of Paper I, the polluters that are able to provide the proper material for the second stellar generation consist of stars with initial masses $\geq 20 M_{\odot}$. We assume that all the low-speed material ejected by those massive stars (at break-up during the main sequence and during the latter $\Omega \Gamma$-limit phase) is used locally to form the second stellar generation after having been diluted to various degrees with pristine ISM. We do not make any assumption regarding the composition of the stellar ejecta, but rather use our theoretical predictions for the composition of the slow wind ${ }^{7}$.

7 In PC06 the estimation of the polluter IMF for NGC 2808 was based on very conservative assumptions that maximised the mass of

\subsection{Number ratio of normal to anomalous stars}

The first quantity we need to determine is the number ratio of second generation (anomalous) stars to the first generation (normal) ones.

If the initial total mass of first generation stars of all masses is $M_{\mathrm{GC}}^{1 \mathrm{G}}$, then the present day mass in first generation long-lived stars is:

$M_{\mathrm{LL}}^{1 \mathrm{G}}=M_{\mathrm{GC}}^{1 \mathrm{G}} \times f_{\mathrm{LL}}^{1 \mathrm{G}} \times\left(1-e_{\mathrm{LL}}^{1 \mathrm{G}}\right)$.

$f_{\mathrm{LL}}^{1 \mathrm{G}}$ is the mass fraction locked initially in first generation longlived stars; it is given by integration over the IMF in the mass range $0.1-0.8 M_{\odot} . e_{\mathrm{LL}}^{1 \mathrm{G}}$ is the fraction of first generation longlived stars that have escaped from the cluster over its history, and reflects the dynamical properties of the cluster as a whole. For the sake of simplicity we assume that this latter parameter is independent of the initial stellar mass.

Regarding the second generation long-lived stars, we must consider the fact that they form out of the slow wind of first generation massive stars that is diluted with pristine gas. The present-day mass locked into those stars is then:

$M_{\mathrm{LL}}^{2 \mathrm{G}}=M_{\mathrm{GC}}^{1 \mathrm{G}} \times f_{\mathrm{SW}} \times(1+d) \times f_{\mathrm{LL}}^{2 \mathrm{G}} \times\left(1-e_{\mathrm{LL}}^{2 \mathrm{G}}\right)$.

$f_{\mathrm{SW}}$ is the mass fraction of slow winds produced by massive stars, which is given by theoretical stellar models. $d$ is the parameter reflecting global dilution with pristine ISM; it can be inferred from detailed analysis of the observed abundance patterns, as discussed in Sect. 5.5. $f_{\mathrm{LL}}^{2 \mathrm{G}}$ and $e_{\mathrm{LL}}^{2 \mathrm{G}}$ are respectively the initial mass fraction of the second generation long-lived stars and the fraction of these objects that have escaped the cluster (as for $e_{\mathrm{LL}}^{1 \mathrm{G}}$ we assume that $e_{\mathrm{LL}}^{2 \mathrm{G}}$ is independent of the initial stellar mass).

Therefore, the number ratio between these two populations of long-lived stars is:

$\frac{n_{\mathrm{LL}}^{2 \mathrm{G}}}{n_{\mathrm{LL}}^{1 \mathrm{G}}}=\frac{\left\langle M_{\mathrm{LL}}^{1 \mathrm{G}}\right\rangle}{\left\langle M_{\mathrm{LL}}^{2 \mathrm{G}}\right\rangle} \times f_{\mathrm{SW}} \times(1+d) \times \frac{f_{\mathrm{LL}}^{2 \mathrm{G}}}{f_{\mathrm{LL}}^{1 \mathrm{G}}} \times \frac{1-e_{\mathrm{LL}}^{2 \mathrm{G}}}{1-e_{\mathrm{LL}}^{1 \mathrm{G}}}$,

where $\left\langle M_{\mathrm{LL}}^{1 \mathrm{G}}\right\rangle$ and $\left\langle M_{\mathrm{LL}}^{2 \mathrm{G}}\right\rangle$ are the average masses of the long-lived stars of first and second generation respectively. The left-hand part of this equation can be inferred from observational studies, following the method proposed by PC06 and based on the observed $[\mathrm{O} / \mathrm{Na}]$ distribution; this point is discussed further in Sect. 5.6. The number of escaping stars is a free parameter that is related to the cluster dynamics.

The stellar IMF of both generations influences the quantities $f_{\mathrm{SW}}, f_{\mathrm{LL}}^{1 \mathrm{G}}$, and $f_{\mathrm{LL}}^{2 \mathrm{G}}$, as well as the ratio $\left\langle M_{\mathrm{LL}}^{1 \mathrm{G}}\right\rangle /\left\langle M_{\mathrm{LL}}^{2 \mathrm{G}}\right\rangle$. We follow PC06 and assume that the IMF of the globular cluster is composite. For stars of first generation more massive than $0.8 M_{\odot}$, the IMF is approximated by a power-law of the form:

$\Phi(M)=\frac{\mathrm{d} N}{\mathrm{~d} M} \propto M^{-(1+x)}$,

with $x$ the slope that has to be determined ( $x=1.35$ being the Salpeter 1955 value). On the other hand we consider that the present-day log-normal distribution derived by Paresce \& De Marchi (2000) reflects the IMF of long-lived stars (0.1$0.8 M_{\odot}$ ) of both first and second generations:

$\ln \Phi(M) \propto A-\left(\frac{\log \left(M / M_{\mathrm{C}}\right)}{2 \sigma}\right)^{2}$,

the H-processed ejecta released by massive stars. In particular, all the mass outside the He-core was assumed to have the proper composition. 
where $M_{\mathrm{C}}=0.33 \pm 0.03$ is the peak-mass of the log-normal distribution, $\sigma=0.34 \pm 0.04$ a standard deviation, and $A$ a normalisation constant. We normalise all IMF to unity:

$$
\int_{0.1}^{120} M \Phi(M) \mathrm{d} M=1 \text {. }
$$

Since we presume the same IMF for the first and second generation long-lived stars, one has that $\left\langle M_{\mathrm{LL}}^{1 \mathrm{G}}\right\rangle=\left\langle M_{\mathrm{LL}}^{2 \mathrm{G}}\right\rangle$.

\subsection{Chemical composition of second generation stars}

The next step consists of the derivation of the equations required to determine the chemical abundance distribution in the matter out of which the second generation forms. This distribution will reflect directly in the star-to-star abundance variations at their birth. It will also reflect the distribution in the star-to-star abundance variations that we observe today in stars that have not undergone any change of their surface abundances by whatever internal mixing process, i.e. typically for stars having not yet evolved through their first dredge-up ${ }^{8}$. We recall that this matter consists of the slow winds of individual stars of first generation diluted locally and to various degrees with pristine ISM. As discussed in Sect. 2, the degree of enrichment of the slow wind by the H-burning products is likely to be different at the beginning and the end of the evolution of the massive polluter. Let us call $t$ the age of the massive star polluter, then

$X_{i}^{2 \mathrm{G}}\left(a_{t}\right)=\left(1-a_{t}\right) X_{i}^{\mathrm{SW}}(t)+a_{t} X_{i}^{\mathrm{init}}$

with $X_{i}^{\text {init }}$ and $X_{i}^{\mathrm{SW}}$ being the mass fraction of the element $i$ respectively in the pristine ISM and in the slow wind of the polluter. $X_{i}^{2 \mathrm{G}}$ corresponds to the same quantity after dilution of the massive star ejecta with pristine material, $a_{t}$ being the local dilution parameter (see more detail in Sect. 5.5).

Let us call $\dot{m}_{\mathrm{SW}}(t, M)$ the rate of ejection of mass in the slow wind at time $t$ by a star of given initial mass $M$. The mass ejected during the time $\mathrm{d} t$ is $\dot{m}_{\mathrm{SW}}(t, M) \mathrm{d} t$. After dilution, the matter available to form the second generation stars from this mass is $\dot{m}_{\mathrm{SW}}(t) \mathrm{d} t /\left(1-a_{t}\right)$. Integrating over the duration of the "slow wind" phase, $\Delta t$ and over the IMF, one finds an expression for the matter available to form the second generation stars,

$M_{\mathrm{GC}}^{2 \mathrm{G}}=M_{\mathrm{GC}}^{1 \mathrm{G}} \int_{20}^{120}\left(\int_{\Delta t} \dot{m}_{\mathrm{SW}}(t, M) \frac{1}{1-a_{t}} \mathrm{~d} t\right) \Phi(M) \mathrm{d} M$.

In this case, the present-day mass locked into these stars is then

$M_{\mathrm{LL}}^{2 \mathrm{G}}=M_{\mathrm{GC}}^{2 \mathrm{G}} \times f_{\mathrm{LL}}^{2 \mathrm{G}} \times\left(1-e_{\mathrm{LL}}^{2 \mathrm{G}}\right)$.

By identification between Eqs. (9) and (2) we can obtain a relation between the global dilution parameter $d$ and the local one $a_{t}$ :

$d=\frac{1}{f_{\mathrm{SW}}} \int_{20}^{120} \int_{\Delta t} \dot{m}_{\mathrm{SW}}(t, M) \frac{a_{t}}{1-a_{t}} \mathrm{~d} t \Phi(M) \mathrm{d} M$.

In order to obtain the mass available to form second generation stars with a given abundance of the element $i$ equal to $X_{i}^{2 \mathrm{G}}$, the integral over time in Eq. (8) has to be restrained to that portion of the slow wind phase, during which the wind has a given abundance of the element $i$ equal to $X_{i}^{\mathrm{SW}}$, where $X_{i}^{\mathrm{SW}}$ is the abundance such that, after dilution, the material made of slow wind and ISM

8 These stars may however have suffered from some depletion of their lithium surface abundance. will have an abundance equal to $X_{i}^{2 \mathrm{G}}$. If we call $\Delta t_{i}$ this duration, then, the mass available to form second generation stars with a given abundance of the element $i$ equal to $X_{i}^{2 \mathrm{G}}$ is given by

$$
M_{\mathrm{GC}}^{2 \mathrm{G}}\left(X_{i}^{2 \mathrm{G}}\right)=M_{\mathrm{GC}}^{1 \mathrm{G}} \int_{20}^{120}\left(\int_{\Delta t_{i}} \dot{m}_{\mathrm{SW}}(t, M) \frac{1}{1-a_{t}} \mathrm{~d} t\right) \Phi(M) \mathrm{d} M,
$$

and the present-day mass locked into those stars is then

$$
M_{\mathrm{LL}}^{2 \mathrm{G}}\left(X_{i}^{2 \mathrm{G}}\right)=M_{\mathrm{GC}}^{2 \mathrm{G}}\left(X_{i}^{2 \mathrm{G}}\right) \times f_{\mathrm{LL}}^{2 \mathrm{G}} \times\left(1-e_{\mathrm{LL}}^{2 \mathrm{G}}\right) \text {. }
$$

Normalised distribution is obtained by dividing Eq. (12) by the total number, $M_{\mathrm{LL}}$, of first and second generation stars given respectively by Eqs. (1) and (2):

$$
\frac{M_{\mathrm{LL}}^{2 \mathrm{G}}\left(X_{i}^{2 \mathrm{G}}\right)}{M_{\mathrm{LL}}}=\frac{M_{\mathrm{GC}}^{2 \mathrm{G}}\left(X_{i}^{2 \mathrm{G}}\right) \times f_{\mathrm{LL}}^{2 \mathrm{G}} \times\left(1-e_{\mathrm{LL}}^{2 \mathrm{G}}\right)}{f_{\mathrm{LL}}^{1 \mathrm{G}}\left(1-e_{\mathrm{LL}}^{1 \mathrm{G}}\right)+f_{\mathrm{SW}}(1+d) f_{\mathrm{LL}}^{2 \mathrm{G}}\left(1-e_{\mathrm{LL}}^{2 \mathrm{G}}\right)}
$$

Note that the above mass ratio is also equal to a number ratio provided the mean mass of the low mass stars in the first and second generation are the same, which is the hypothesis we have made here.

We will use the theoretical predictions of our models of fast rotating massive stars published in Paper I. In particular, we have that the stars with initial masses of $20,40,60$, and $120 M_{\odot}$ expel respectively $1.7,12.5,24.3$, and $48.0 M_{\odot}$ in total in their slow wind. In order to convolve by the polluter IMF we interpolate between those values for polluter stars with different initial masses.

\subsection{Mass of stellar remnants}

Another important quantity is the contribution of stellar remnants (i.e., white dwarfs, neutron stars or stellar black holes) to the total cluster mass. Indeed first and second generation stars more massive than the so-called long-lived stars (i.e., with initial masses higher than $0.8 M_{\odot}$ ) already died and produced dark remnants ${ }^{9}$. We call $f_{\text {rem }}^{1 \mathrm{G}}$ and $f_{\text {rem }}^{2 \mathrm{G}}$ the mass fraction to the total stellar mass of respectively first and second generation remnants:

$f_{\mathrm{rem}}=\int_{0.8}^{120} R(M) \Phi(M) \mathrm{d} M$.

We follow PC06 for the relations between the initial mass of a star and the mass of its remnant:

$R(M)= \begin{cases}0.446+0.106 M & \text { for } M<10 M_{\odot} \\ 1.5 & \text { for } 10<M<25 M_{\odot} \\ 3 & \text { for } M>25 M_{\odot} .\end{cases}$

The total mass of the remnants in the cluster today is given by:

$M_{\mathrm{rem}}=M_{\mathrm{GC}}^{1 \mathrm{G}} f_{\mathrm{rem}}^{1 \mathrm{G}}\left(1-e_{\mathrm{rem}}^{1 \mathrm{G}}\right)+M_{\mathrm{GC}}^{1 \mathrm{G}} f_{\mathrm{SW}}(1+d) f_{\mathrm{rem}}^{2 \mathrm{G}}\left(1-e_{\mathrm{rem}}^{2 \mathrm{G}}\right)$

with $e_{\mathrm{rem}}^{1 \mathrm{G}}$ and $e_{\mathrm{rem}}^{2 \mathrm{G}}$ the fraction of remnants lost by the cluster during its evolution (these quantities take into account the loss of both remnant progenitors and remnants).

Therefore the mass ratio between remnants and long-lived stars today is obtained by combining Eqs. (1), (2), and (16), i.e.:

$\frac{M_{\mathrm{rem}}}{M_{\mathrm{LL}}}=\frac{f_{\mathrm{rem}}^{1 \mathrm{G}}\left(1-e_{\mathrm{rem}}^{1 \mathrm{G}}\right)+f_{\mathrm{SW}}(1+d) f_{\mathrm{rem}}^{2 \mathrm{G}}\left(1-e_{\mathrm{rem}}^{2 \mathrm{G}}\right)}{f_{\mathrm{LL}}^{1 \mathrm{G}}\left(1-e_{\mathrm{LL}}^{1 \mathrm{G}}\right)+f_{\mathrm{SW}}(1+d) f_{\mathrm{LL}}^{2 \mathrm{G}}\left(1-e_{\mathrm{LL}}^{2 \mathrm{G}}\right)}$

\footnotetext{
9 As mentioned in Sect. 3.2, we will assume later that only long-lived stars formed in the second generation. However we derive here the most general equations.
} 


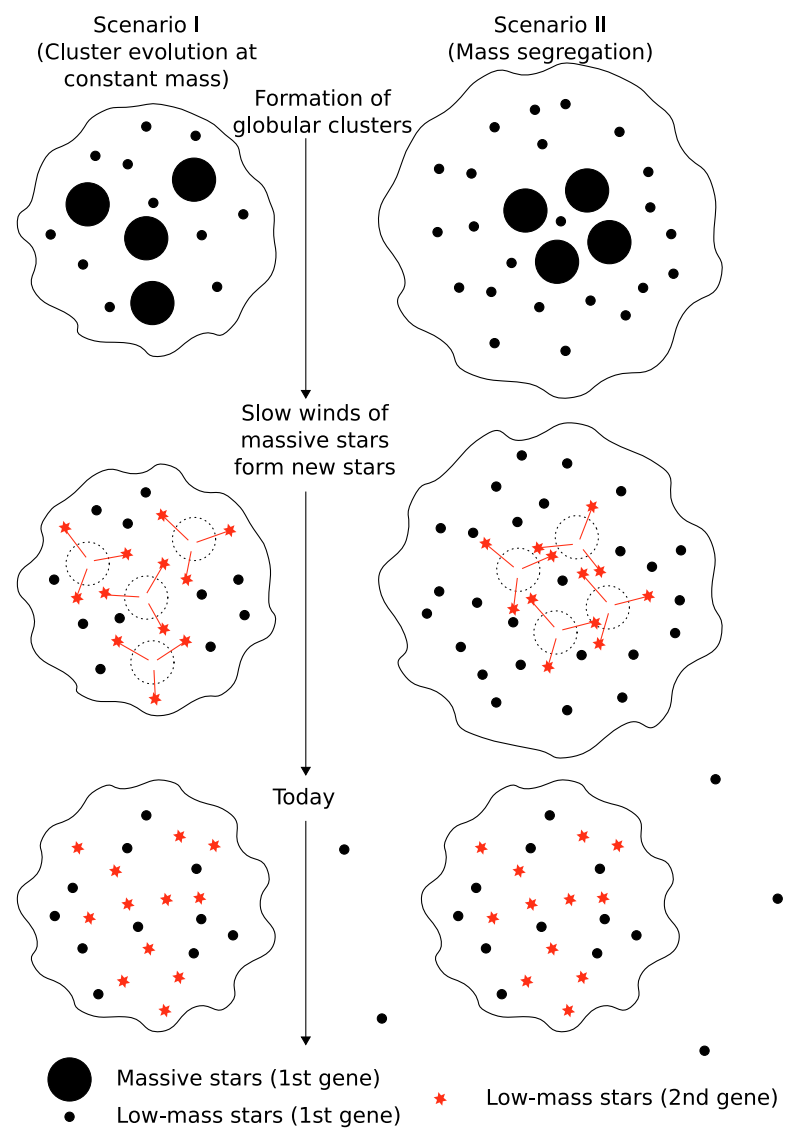

Fig. 2. Schematic illustration of the scenarios discussed in Sect. 4. Black circles and red stars stand for first and second generation stars respectively. In the middle panels, the dashed symbols represent fast evolving massive stars giving birth to second generation stars. In both cases, only long-lived low-mass stars form in the second generation. In Scenario I (left panels) all the stars remain in the cluster and the results depend on the chosen IMF. In Scenario II (right panels), we assume mass segregation and pollution occurs only at the center of the cluster; the first generation long-lived stars that remained in the outer part are assumed to be lost over the whole dynamical evolution.

\section{Our two scenarios for the dynamical evolution of the cluster}

As some of the parameters used in the previous equations are poorly known and are related to general properties of globular clusters (i.e., their IMF and their dynamical evolution) that are poorly constrained, we explore two simplifying scenarios. For the sake of simplicity, we make the following assumptions in both cases. We suppose that the first stellar generation contains stars with initial masses between 0.1 and $120 M_{\odot}$. Regarding the second generation, we assume that it consisted only of lowmass long-lived stars (i.e., with an initial mass between 0.1 and $0.8 M_{\odot}$ ); this latter assumption will obviously minimise the constraint on the polluter IMF.

\subsection{Scenario I: no loss of stars and of stellar remnants}

The first scenario we consider (left panels in Fig. 2) assumes that the cluster as a whole has retained all its stars and stellar remnants. In other words, the cluster stellar component underwent no evaporation of stars due for instance to tidal stripping. In this case one has thus

$e_{\mathrm{LL}}^{1 \mathrm{G}}=e_{\mathrm{LL}}^{2 \mathrm{G}}=e_{\mathrm{rem}}^{1 \mathrm{G}}=e_{\mathrm{rem}}^{2 \mathrm{G}}=0$.
Since we assume that only low-mass stars are produced in the second generation, we have

$f_{\mathrm{LL}}^{2 \mathrm{G}}=1$ and $f_{\mathrm{rem}}^{2 \mathrm{G}}=0$.

We also assume that the slope of the IMF is the same as far as the first and second generation long-lived stars are concerned. As a result of these simplifications, the general equations derived in Sect. 3 become

$\frac{n_{\mathrm{LL}}^{2 \mathrm{G}}}{n_{\mathrm{LL}}^{1 \mathrm{G}}}=\frac{f_{\mathrm{SW}}(1+d)}{f_{\mathrm{LL}}^{1 \mathrm{G}}}$,

for the number ratio between normal and anomalous long-lived stars (see Eq. (3)),

$\frac{M_{\mathrm{LL}}^{2 \mathrm{G}}\left(X_{i}^{2 \mathrm{G}}\right)}{M_{\mathrm{LL}}}=\frac{\int_{20}^{120}\left(\int_{\Delta t_{i}} \dot{m}_{\mathrm{SW}}(t, M) \frac{1}{1-a_{t}} \mathrm{~d} t\right) \Phi(M) \mathrm{d} M}{f_{\mathrm{LL}}^{1 \mathrm{G}}+f_{\mathrm{SW}}(1+d)}$,

for the number fraction of stars of second generation with a given composition (see Eq. (13)), and

$\frac{M_{\mathrm{rem}}}{M_{\mathrm{LL}}}=\frac{f_{\mathrm{rem}}^{1 \mathrm{G}}}{f_{\mathrm{LL}}^{1 \mathrm{G}}+f_{\mathrm{SW}}(1+d)}$

for the fraction of the total mass in stellar remnants (see Eq. (17)).

The free parameters in Scenario I are the slope of the IMF of the polluters and the dilution factor. It should be noted that the Scenario I with no evaporation of stars is equivalent to that derived in the case where stars of both first and second generation leave the cluster in a similar way (i.e. where $e_{\mathrm{LL}}^{1 \mathrm{G}}=e_{\mathrm{LL}}^{2 \mathrm{G}}=e_{\mathrm{rem}}^{1 \mathrm{G}}=$ $e_{\text {rem }}^{2 \mathrm{G}}$ but are not necessarily equal to 0 as in Eq. (18)).

\subsection{Scenario II: mass segregation and evaporation of stars}

In Scenario II, we assume (see right panels in Fig. 2) that the massive polluters of first generation were born in the center of the cluster or have migrated very rapidly towards this region. In this case, the second generation stars are created only in the central region while the external part of the cluster hosts only first generation long-lived low-mass stars. This strong radial distribution is assumed to stay in place until the moment where supernovae sweep away the residual intra-cluster gas and thus strongly modify the cluster potential well. As a consequence, stars in the external part of the cluster do not remain bound and are ejected into the galactic halo.

In this case, we impose a Salpeter IMF for the polluters ${ }^{10}$, and search for the amount of first generation long-lived stars that need to be lost in order to fit the observational constraints. As a result of our hypothesis, one has $f_{\mathrm{LL}}^{2 \mathrm{G}}=1, e_{\mathrm{LL}}^{2 \mathrm{G}}=0$, while $e_{\mathrm{LL}}^{1 \mathrm{G}}$ remains a free parameter. The general equations become (see Eqs. (3), (13) and (17)):

$\frac{n_{\mathrm{LL}}^{2 \mathrm{G}}}{n_{\mathrm{LL}}^{1 \mathrm{G}}}=\frac{f_{\mathrm{SW}}(1+d)}{f_{\mathrm{LL}}^{1 \mathrm{G}}\left(1-e_{\mathrm{LL}}^{1 \mathrm{G}}\right)}$,

$\frac{M_{\mathrm{LL}}^{2 \mathrm{G}}\left(X_{i}^{2 \mathrm{G}}\right)}{M_{\mathrm{LL}}}=\frac{\int_{20}^{120} \int_{\Delta t_{i}} \dot{m}_{\mathrm{SW}}(t, M) \frac{1}{1-a_{t}} \mathrm{~d} t \Phi(M) \mathrm{d} M}{f_{\mathrm{LL}}^{1 \mathrm{G}}\left(1-e_{\mathrm{LL}}^{1 \mathrm{G}}\right)+f_{\mathrm{SW}}(1+d)}$,

${ }^{10}$ It should be noted that this IMF is not valid for the whole mass range of polluters. However our findings would not change significantly with a more realistic IMF as that of Kroupa et al. (1993). 


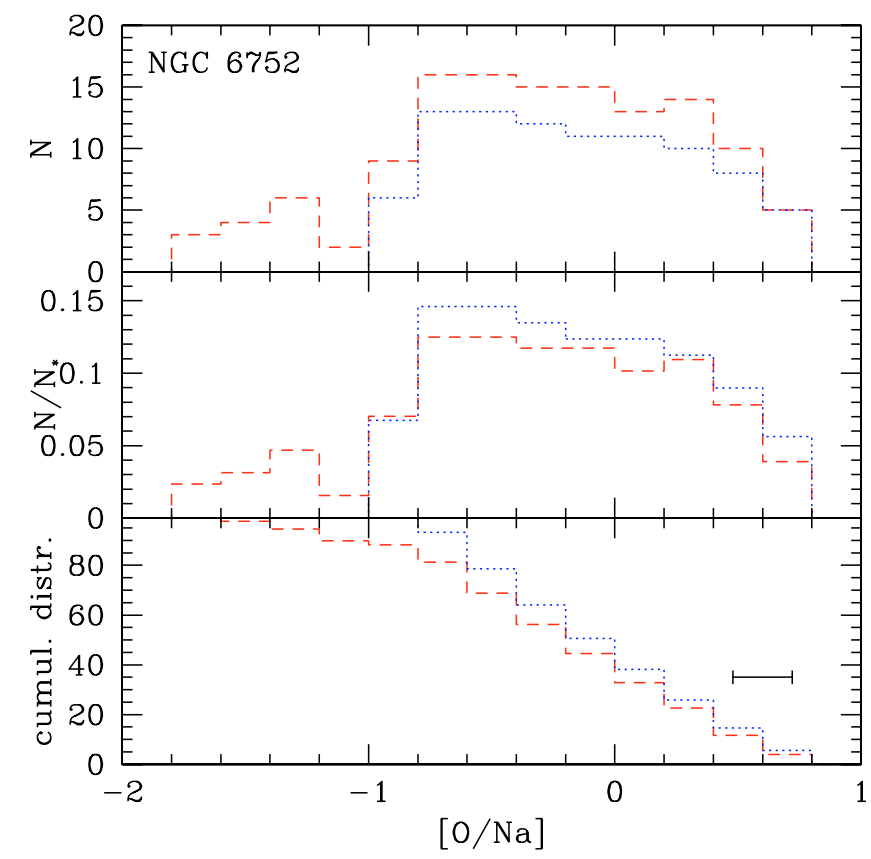

Fig. 3. ONa distribution function in NGC 6752 based on observations of red giant branch stars from Carretta et al. (2007a). (Top) Distribution function in a number issue taken from Carretta et al. (2007a); (middle) normalised distribution function, $N_{*}$ is the total number of observed stars; (bottom) inverse cumulative distribution function for the same stars. Average $[\mathrm{O} / \mathrm{Na}]$ error is also indicated. Dotted lines refer to stars for which $\mathrm{O}$ and $\mathrm{Na}$ abundances have been determined. A sample of stars have determinations of the $\mathrm{Na}$ abundance but not of $\mathrm{O}$. In this case the abundance of $\mathrm{O}$ has been inferred from the $\mathrm{Na}$ abundance using the global anticorrelation between these two elements. Inclusion of this sample of stars gives the distributions shown by the dashed lines.

and

$$
\frac{M_{\mathrm{rem}}}{M_{\mathrm{LL}}}=\frac{f_{\mathrm{rem}}^{1 \mathrm{G}}\left(1-e_{\mathrm{rem}}^{1 \mathrm{G}}\right)}{f_{\mathrm{LL}}^{1 \mathrm{G}}\left(1-e_{\mathrm{LL}}^{1 \mathrm{G}}\right)+f_{\mathrm{SW}}(1+d)} .
$$

The free parameters in Scenario II are the number fractions of stars lost by the clusters and the dilution factor.

\section{The case of NGC 6752}

Armed with these equations, we will first focus on the globular cluster NGC 6752 which has about the same metallicity $([\mathrm{Fe} / \mathrm{H}] \sim-1.56$, Carretta et al. 2007a) and initial abundance pattern as the stellar models presented in Paper I. Our theoretical nucleosynthetic predictions can thus be directly applied. Additionally, NGC 6752 is the Galactic globular cluster with the largest set of abundance data for numerous chemical elements (see references in Paper I).

\subsection{Observed ratio of anomalous to normal stars in NGC 6752}

To determine the ratio between the first generation normal and second generation anomalous long-lived stars in NGC 6752, we use the method described in PC06 and base our estimation on the $[\mathrm{O} / \mathrm{Na}]$ distribution function derived from the observations by Carretta et al. (2007a, see Fig. 3). Typical dispersion errors on $\mathrm{O}$ and $\mathrm{Na}$ abundances in the sample of 120 stars are respectively $\sigma_{\mathrm{O}}=0.101 \mathrm{dex}$ and $\sigma_{\mathrm{Na}}=0.060$ dex. The total dispersion on

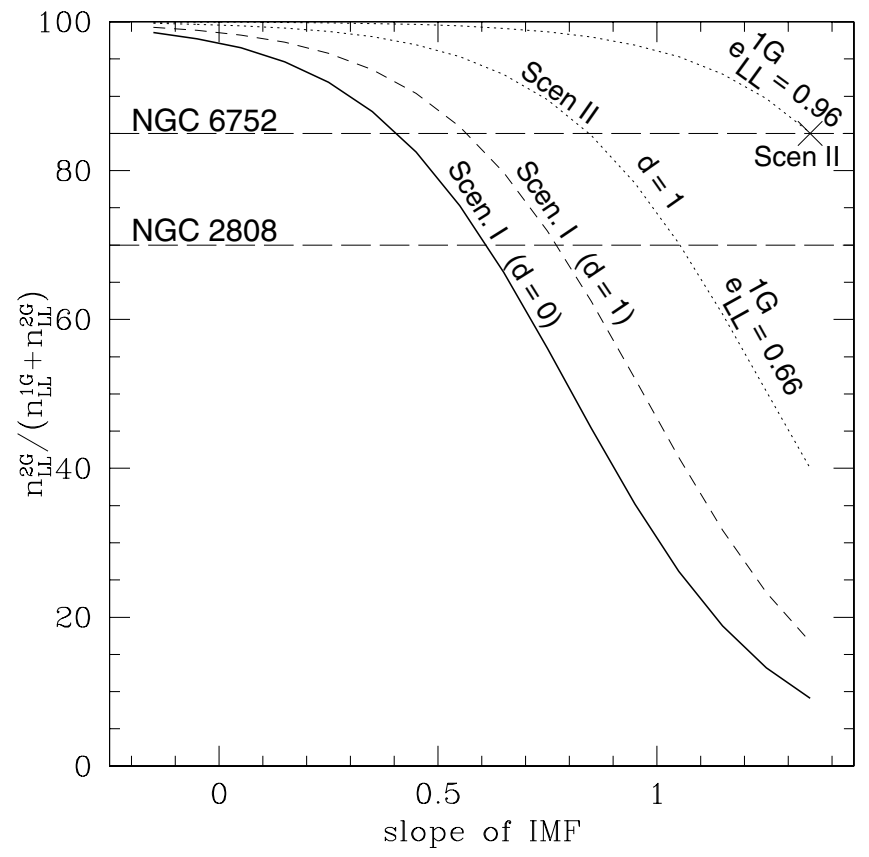

Fig. 4. Percentage of second generation stars in the total long-lived stars observed today as a function of the IMF slope of the first generation polluters. The solid line refers to long-lived stars born out of the slow winds of massive stars only, while the dashed line corresponds to the case where dilution of the ejecta with pristine ISM is taken into account with a global dilution factor $d=1$. Dotted lines show the cases where $d=1, e_{\mathrm{LL}}^{1 \mathrm{G}}=0.66$ and 0.96 . The cross indicates the point corresponding to a Salpeter IMF and to $85 \%$ of second generation longlived stars. Horizontal lines indicate the constraints coming from the observed $[\mathrm{O} / \mathrm{Na}]$ distribution for two well-studied Galactic globular clusters.

the $[\mathrm{O} / \mathrm{Na}]$ ratio is thus $\sigma_{\mathrm{ONa}}=\sqrt{\sigma_{\mathrm{O}}^{2}+\sigma_{\mathrm{Na}}^{2}}=0.117$, so that the first generation stars span a range of $\Delta[\mathrm{O} / \mathrm{Na}]=2 \sigma_{\mathrm{ONa}}=$ 0.234 . As this value is higher than the bin width of 0.2 used for the distribution functions displayed in Fig. 3, first generation stars can be found in the two bins with the highest $[\mathrm{O} / \mathrm{Na}]$ ratio. They represent between $12 \%$ and $14 \%$ of the total number of long-lived stars. In the following, we round up to $85 / 15$ the ratio between second and first generation long-lived stars.

The percentage of first generation long-lived stars we find in NGC 6752 is lower than the values found in previous studies. PC06 find indeed $30 \%$ of normal stars in NGC 2808 while Carretta et al. (2005) infer that less than $1 / 3$ of the stars are normal on the basis of CNO abundances in NGC 6397, NGC 6752, and 47 Tuc. This latter study does not however use very precise statistics as it mixes several clusters. This is discussed further in Sect. 7.1.

\subsection{IMF slope for the polluter stars in Scenario I}

In Scenario I, the number fraction of second to first generation stars (see Eq. (20)) depends on the slope of the IMF of the polluters and on the global dilution factor $d$. Figure 4 displays the fraction of second generation stars as a function of the IMF slope of the polluters and for dilution factors $d=0$ and 1 .

A Salpeter IMF with no differential evaporation leads to a small number of second generation stars that total up 10-17\% of the cluster long-lived stars depending on the dilution factor $(0$ or 1). In order to have $85 \%$ of second generation long-lived stars in the framework of Scenario I, the IMF slope must be around 0.4 
if $d=0$ (full line). This value is a lower limit as it does not take into account dilution of the slow wind ejecta with some pristine gas. For a global dilution parameter $d=1$, the IMF slope raises up to 0.55 (see the dashed line). Estimating in the same way the IMF slope of the polluters for the cluster NGC 2808, which has a higher fraction of normal first generation stars, results in steeper IMF ( 0.60 and 0.76 for respectively $d=0$ and 1$)$, although still quite flat with respect to the Salpeter one. Thus, as already discussed in PC06, Scenario I implies relatively flat IMFs for the polluters.

\subsection{Fraction of stars lost by the cluster in Scenario II}

We consider now Scenario II where we use a Salpeter IMF for first generation massive stars. A dilution factor $d=1$ is used. In order to explain the high number of second generation anomalous stars observed today, a strong loss of first generation longlived stars from the cluster is required. More precisely, we derive $e_{\mathrm{LL}}^{1 \mathrm{G}} \simeq 0.958$ (see the corresponding dotted curve in Fig. 4). This means that, in the present day globular cluster, only $\sim 4.2 \%$ of the first generation long-lived stars are still present. Note that this is a lower limit; indeed, if some second generation stars also escaped in the halo, then even more first generation stars must have been lost by the cluster in order to reproduce the high number of second generation anomalous stars observed today in NGC 6752. It should be noted that the unpolluted pop. II stars found in the halo would comes largely from lower-mass clusters that dissolved after residual gas expulsion, as pointed out by Kroupa \& Boily (2002).

An intermediate case between Scenario I and II is shown in Fig. 4 (dotted line labelled with $e_{\mathrm{LL}}^{1 \mathrm{G}}=0.66$ ). There we assume that one third of first generation long-lived stars remain in the cluster. In this third case, an intermediate IMF slope of 0.85 is needed in order to reproduce the observed ratio of anomalous to normal stars in NGC 6752.

\subsection{Amount of stellar residues}

Figure 5 displays the mass fraction of different stellar populations at birth (i.e. before any evaporation of stars) as a function of the IMF slope for the polluters. Normalisation in made with the total mass of gas used to form stars (i.e., the gas used to form the first stellar generation added to the one used for the dilution process at the birth of anomalous stars). As the IMF slope decreases, massive stars initially dominate the cluster mass; for an IMF of $\sim 0.55$, the matter ejected in the slow winds represents $\sim 20 \%$ of the total mass of all first generation stars and more than three times the mass locked into long lived first generation stars.

The left panel of Fig. 6 shows how the total stellar mass of the present day cluster varies as a function of the polluter IMF in the frame of Scenario I (continuous line). The mass is normalised to the mass of gas used to form stars. The following remarks can be made:

- We see that the stellar component in the present day cluster represents about half of the total mass of gas used to form stars (more precisely between $\sim 47$ and $57 \%$ depending on the slope of the IMF for the polluters). The rest of the mass consists of material ejected by the stars under the form of fast ejecta escaping the potential well of the cluster (fast winds of massive stars, supernova ejecta, winds of red giants and asymptotic giant branch stars).

- The way the total mass varies as a function of the slope of the IMF does appear at first sight quite surprising. Why do

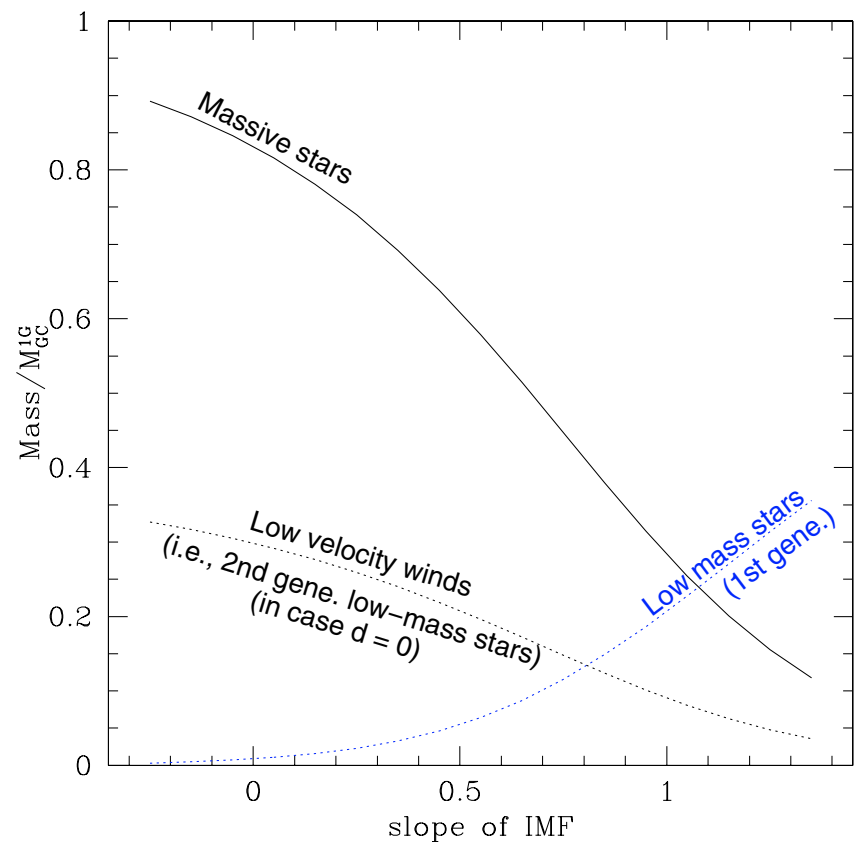

Fig. 5. Mass fractions of different stellar populations at birth normalised to the mass of the first generation stars, as a function of the slope of the polluter IMF. Full line refers to the massive stars $\left(20-120 M_{\odot}\right)$. Dotted lines indicate the long-lived stars $\left(0.1-0.8 M_{\odot}\right)$ of first and second generations (see the labels of the curves). The mass fraction of second generation stars corresponds to the case without dilution.

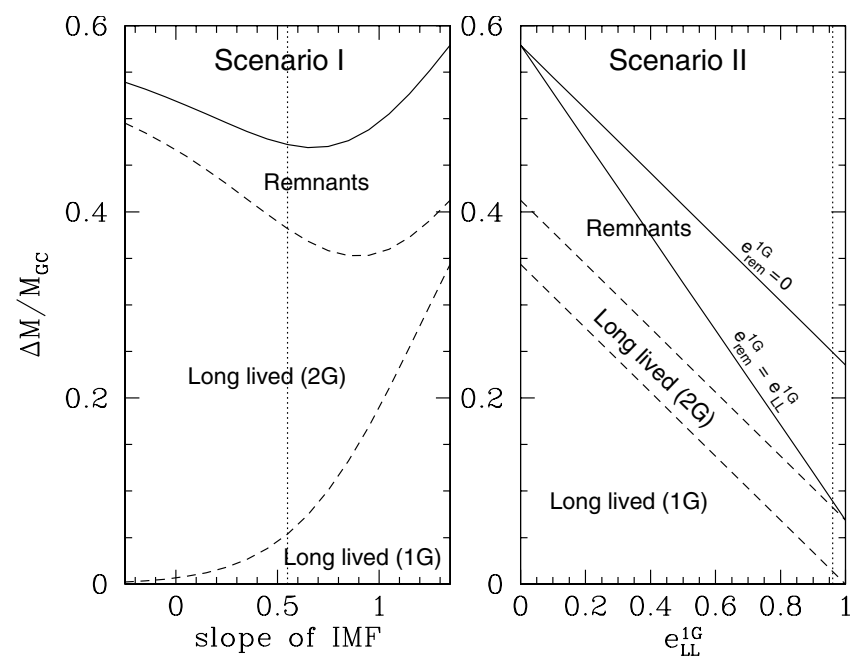

Fig. 6. Variation of the present day total mass of the cluster (continuous line) as a function of the slope of the IMF (Scenario I, left panel) and as a function of the fraction of stars of the first generation which have been lost by the cluster, $e_{\mathrm{LL}}^{\mathrm{IG}}$ (Scenario II, right panel). The vertical extension of each of the three labeled zones gives the mass fraction of the cluster locked into long lived stars of first and second generation stars and in remnants. The line labeled as $e_{\mathrm{rem}}^{1 \mathrm{G}}=0$ and $e_{\mathrm{rem}}^{1 \mathrm{G}}=e_{\mathrm{LL}}^{1 \mathrm{G}}$ indicate respectively the case where no remnant are lost by the clusters and where they have been lost at the same rate as long-lived stars. The vertical dotted line shows the ratio of second to first generation stars is equal to the observed value of $85 / 15$.

we obtain a non-monotonic behaviour? We note that if there had been only a unique star formation episode, the present day mass cluster would be lower than the mass contained in the gas used to form stars. As explained above, the cluster loses fast stellar ejecta. Since their proportion increases when the slope of the IMF is decreased, smaller masses of 
the present day clusters are obtained with flatter IMF. In our case, the situation is not so straightforward, since part of the massive star ejecta are actually released under the form of slow winds which are retained by the clusters and are used to form the second generation stars. Therefore, the increase of the number of massive stars (i.e. a decrease of the slope of the IMF) may produce either a decrease or an increase of the present day mass cluster, depending on how the mass lost by the fast ejecta is compensated by the second generation stars formed from the slow ejecta and from some amount of ISM. From Fig. 6, we see that for an IMF slope inferior to $\sim 0.75$, the mass locked in second generation stars is greater than the mass lost through fast ejecta, while for slopes of the IMF superior to 0.75 , the opposite situation occurs.

- The population of remnants never exceeds those of long lived stars. At most, it can contain about one third of the present day mass in the case of a Salpeter IMF. The vertical line at an abscissa of 0.55 corresponds to a ratio of anomalous (second generation) to normal (first generation) star equal to $85 / 15$.

The right panel of Fig. 6 shows how the present day mass of the cluster varies as a function of the fraction of stars of the first generation which escape the cluster. Since an IMF slope of 1.35 is chosen in that case, we obtain for $e_{\mathrm{LL}}^{1 \mathrm{G}}=0$ the same results as in Scenario I at the corresponding IMF. As already obtained in Fig. 4, we see that to obtain a ratio of anomalous (second generation) to normal (first generation) star equal to 85/15 (see the vertical line with an abscissa equal to 0.958 ), a very large fraction of first generation long lived stars should have been lost by the cluster. For this value of $e_{\mathrm{LL}}^{1 \mathrm{G}}$, in the case that no remnant has escaped $\left(e_{\mathrm{rem}}^{1 \mathrm{G}}=0\right)$, the mass of the cluster would be dominated by the stellar residues. Only when a large fraction of the residues also escape the cluster $\left(e_{\mathrm{rem}}^{1 \mathrm{G}}=e_{\mathrm{LL}}^{1 \mathrm{G}}\right)$, would its mass be dominated by the stars. In that case the present day mass of the cluster represents less than $10 \%$ of the mass of the gas used to form stars.

\subsection{Amount of dilution}

Let us now turn to the dilution that occurs between the slow ejecta of massive stars and pristine gas. If all the ejecta of all potential polluters were fully mixed before the formation of the second generation stars and no dilution with ISM occurs, Eq. (7) should be replaced by:

$X_{i}^{2 \mathrm{G}}=\frac{\int_{20}^{120} \tilde{m}_{\mathrm{SW}} \tilde{X}_{i} \Phi(M) \mathrm{d} M}{\int_{20}^{120} \tilde{m}_{\mathrm{SW}} \Phi(M) \mathrm{d} M}$,

with, for a given individual star $\tilde{m}_{\mathrm{SW}}$ and $\tilde{X}_{i}$ being respectively the total mass ejected in its slow wind and the mean mass fraction of a given element $i$ in its ejecta. In that case second generation stars would all have the same composition that is given in Table 1 for the two extreme IMF slopes found previously (i.e., 0.4 and 1.35$)^{11}$. If we take this fully mixed material and then dilute it in a stochastic way with pristine gas, one can form second generation stars with composition ranging between that of

\footnotetext{
${ }^{11}$ Note that the ejecta composition is weighted by pristine matter released by the polluter at the beginning of the main sequence, when rotational-mixing did not have the time to convey H-burning products from the convective core to the surface. For this reason the 60 and $120 M_{\odot}$ stars eject half of their slow winds with only little abundance variations. This explains the very similar values given in Table 1 for the two different IMF slopes.
}

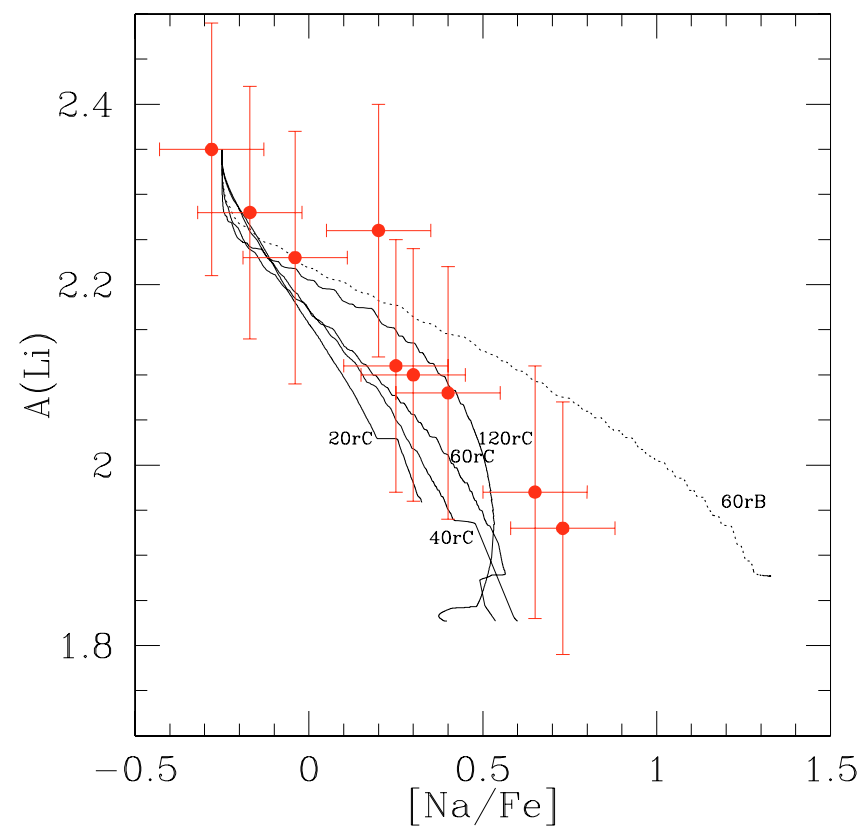

Fig. 7. Li depletion vs. $[\mathrm{Na} / \mathrm{Fe}]$ value. Full circles indicate the observations of turnoff stars by Pasquini et al. (2005) in NGC 6752. The theoretical predictions for the $\mathrm{Li}$ abundance (full and dotted lines) have been decreased by 0.3 dex to take into account in-situ Li depletion during the main sequence of low-mass stars (see PC06 and Charbonnel \& Primas 2005, for more details). Full lines refer to $\mathrm{Li}$ and $\mathrm{Na}$ in the ejecta of individual fast rotating massive stars computed in Paper I; the label indicates the corresponding initial stellar mass. The dotted line corresponds to ejecta of a $60 M_{\odot}$ stars computed with nominal reactions rates (model 60rB in Decressin et al. 2007). We use the minimum dilution factor $a_{\min }=0.3$.

Table 1. Pristine composition and mean composition of slow winds after convolution by an IMF for some elements, He is in mass fraction.

\begin{tabular}{cccc}
\hline \hline Element & Initial & \multicolumn{2}{c}{ IMF slope $(x)$} \\
& & 0.4 & 1.35 \\
\hline$[\mathrm{Na} / \mathrm{Fe}]$ & -0.28 & 0.47 & 0.44 \\
{$[\mathrm{O} / \mathrm{Fe}]$} & 0.32 & 0.06 & 0.10 \\
{$[\mathrm{~N} / \mathrm{Fe}]$} & -0.18 & 0.99 & 0.94 \\
{$[\mathrm{C} / \mathrm{Fe}]$} & -0.16 & -0.56 & -0.53 \\
$A(\mathrm{Li})$ & 2.6 & $-\infty$ & $-\infty$ \\
$\mathrm{He}$ & 0.245 & 0.40 & 0.37 \\
\hline
\end{tabular}

pristine matter (in the case of very strong dilution) and that of the mixed ejecta (in the case without dilution). Only a small range of abundance variations ${ }^{12}$ can be achieved in this way. In this case, the theoretical $[\mathrm{O} / \mathrm{Fe}]$ ratios vary by only $0.26 \mathrm{dex}$, whereas the observed $[\mathrm{O} / \mathrm{Fe}]$ abundances span more than 1 dex in NGC 6752 (Carretta et al. 2007a).

As discussed in Sect. 2, there is additional compelling evidence that the slow wind of an individual star pollutes only on a small scale around its stellar progenitor, and that it is diluted locally with pristine ISM. In order to constrain quantitatively this local dilution process we use the $\mathrm{Li}$ variation detected in NGC 6752 by Pasquini et al. (2005) (see Fig. 7). It is important to recall that the massive star ejecta are totally "Li-free", as this fragile element is destroyed in these objects. We suppose that the matter ejected by an individual massive stars early on the main

\footnotetext{
12 We use the two standard spectroscopic notations: $[\mathrm{X} / \mathrm{Fe}]=$ $\log (\mathrm{X} / \mathrm{Fe})-\log \left(\mathrm{X}_{\odot} / \mathrm{Fe}_{\odot}\right)$, and $A(\mathrm{X})=\log (\mathrm{X} / \mathrm{H})+12$ with $\mathrm{X}, \mathrm{Fe}$, and $\mathrm{H}$ in number density.
} 
sequence encounters more pristine gas and is more diluted than the matter ejected later. As the winds are more and more enriched in $\mathrm{Na}$ in the course of the evolution of a massive polluter, we expect an anticorrelation between $\mathrm{Li}$ and $\mathrm{Na}$ : first, $\mathrm{Li}$-free matter ejected with a low (i.e., close to initial) Na content is more diluted in pristine matter which is Li-rich; low-mass stars with a relatively high Li content and a relatively low $\mathrm{Na}$ abundance are then created. Later, Li-free matter ejected with high $\mathrm{Na}$ abundance is diluted with less pristine material; as a consequence, the newly formed stars have less $\mathrm{Li}$ while their $\mathrm{Na}$ is high. The Li-Na anticorrelation observed by Pasquini et al. (2005) thus provides a calibration of the dilution factor $a$ in Eq. (7). In this paper we use the following expression for this parameter:

$a_{t}=1-\left(1-a_{\min }\right) \frac{t}{t_{*}}$

with $t$ and $t_{*}$ respectively the current time and the total lifetime during which low-velocity winds are ejected. $a_{\text {min }}$ indicates the lower value for the dilution and $a$ decreases from 1 to $a_{\min }$ when the polluter star evolves.

Since $\mathrm{Li}$ is destroyed in massive stars, we have that

$X_{\mathrm{Li}}^{2 \mathrm{G}}\left(a_{t}\right)=a_{t} X_{\mathrm{Li}}^{\mathrm{init}}$,

where $X_{\mathrm{Li}}^{2 \mathrm{G}}$ is the mass fraction of $\mathrm{Li}$ in second generation stars at birth, $X_{\mathrm{Li}}^{\text {init }}$ is the value derived from WMAP data and the standard Big Bang nucleosynthesis $(A(\mathrm{Li})=2.65$ according to Steigman 2006). To take into account the Li depletion (of about 0.3 dex) occurring in low-mass stars (see Charbonnel \& Primas 2005 ), the values of $X_{\mathrm{Li}}^{2 \mathrm{G}}$ are lowered by $0.3 \mathrm{dex}$.

In Fig. 7 we superimpose our theoretical tracks for the Li-Na anticorrelation obtained with a value of $a_{\min }$ equal to 0.3 on the observed anticorrelation determined by Pasquini et al. (2005) in NGC 6752. All the tracks of massive stars show a clear Li-Na anticorrelation with a small dispersion between those tracks (see the continuous lines in Fig. 7).

The anticorrelation follows the same trend in our models and in turnoff stars observed by Pasquini et al. (2005) in NGC 6752. A dilution factor, $a_{\mathrm{min}}$, around 0.3 is needed to reproduce the stars with the lowest Li abundance. An overall agreement (within the observational errors) is obtained with this value as shown in Fig. 7. However it should be noted that uncertainties on the used nuclear reaction rates can modify this anticorrelation. To illustrate this point we show in Fig. 7 the wind composition of a $60 M_{\odot}$ rotating star computed with a different set of reaction rates (dotted line, model 60rB in Paper I). In this case, the $\mathrm{Na}$ enrichment largely exceeds the observed values for the same dilution factor. Thus the uncertainties about the nuclear reaction rates prevent firmer conclusions from being reached.

With the above prescription for $a_{t}$ we can use Eq. (10) to compute the global dilution factor $d$. We find that $d \simeq 1.15$ for both scenarios. The previous findings on the mass budget obtained with $d=1$ are thus still valid.

\subsection{Theoretical ONa distribution}

We compute the theoretical $[\mathrm{O} / \mathrm{Na}]$ distribution function with the parameters we have set up previously for Scenarios I and II (see Eqs. (21) and (24)). A variable dilution factor $a_{t}$ is used with the parameter $a_{\min }=0.3$ (see Eq. (27)). Our results are gathered in Fig. 8 which displays the $[\mathrm{O} / \mathrm{Na}]$ distribution function. First generation stars representing $15 \%$ of the present day distribution are depicted in the hatched area in Fig. 8.

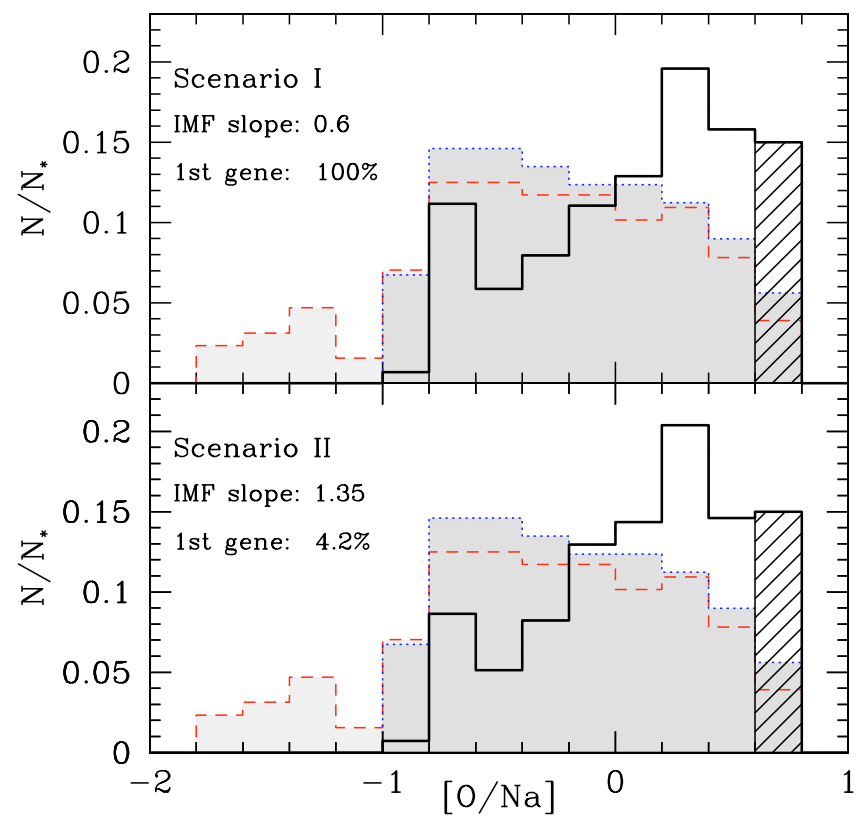

Fig. 8. Distribution function of $[\mathrm{O} / \mathrm{Na}]$ obtained in Scenario I (top) and II (bottom) shown as thick lines. Variable dilution factor with $a_{\min }=0.3$ is taken into account (see Eq. (27)). The label "1st gene" indicates the percentage of first generation long-lived stars remaining in the cluster throughout its evolution. Hatched areas indicate the first generation or "normal" stars. We also superimpose the observed distribution histogram in NGC 6752 (Carretta et al. 2007a) indicated with the dotted and dashed thin lines which have the same meaning as in Fig. 3.

Comparing the two upper panels of Fig. 8, we see that the distributions are strikingly similar. How are two such different scenario possible? We first note that, by construction, the number ratio of anomalous to normal stars is the same in the two scenarios (the parameters have been chosen in order to obtain the same ratio of 85/15). One can make this ratio appear in the denominator of Eqs. (21) and (24) putting in evidence $f_{\mathrm{SW}}(1+d)$. Doing this we see that whatever the scenario considered, the distribution is proportional to the ratio of two quantities involving only masses released by slow winds. A decrease of the slope of the IMF thus produces a similar increase of both the numerator and the denominator.

The peak in the distribution around $[\mathrm{O} / \mathrm{Na}] \sim-0.1$ is due to the stars in the mass range $20-40 M_{\odot}$ which have a longer evolution before reaching the break-up, so that they are more mixed when they release their slow winds.

The dilution with pristine gas adds to the slow winds material characterised with high values of $[\mathrm{O} / \mathrm{Na}]$, therefore dilution increases the values of $[\mathrm{O} / \mathrm{Na}]$. With a $a_{\min }$ parameter of 0.3 no second generation stars can be produced with a $[\mathrm{O} / \mathrm{Na}]$ ratio lower than -1 whereas large amounts of winds generated by the 60 and $120 M_{\odot}$ have a $[\mathrm{O} / \mathrm{Na}]$ ratio lower than -1. NGC 6752 can possibly harbour a population of super-O-poor only detected with a strong $\mathrm{Na}$ abundance (see the dashed line in Fig. 8). For those stars the $\mathrm{O}$ abundance is not directly measured but indirectly determined through the measure of the $\mathrm{Na}$ abundance and using the Na-O anticorrelation. If this group is real we need to change the minimal amount for the mixing to $a_{\min } \simeq 0.03$. But in that case, very low abundances of $\mathrm{Li}$ are expected. At the moment no Li measurements have been performed at the surface of non-evolved stars with such a high $\mathrm{Na}$ content. If feasible, such a measurement would represent an interesting extension of the anticorrelation observed by Pasquini et al. (2005). Another 


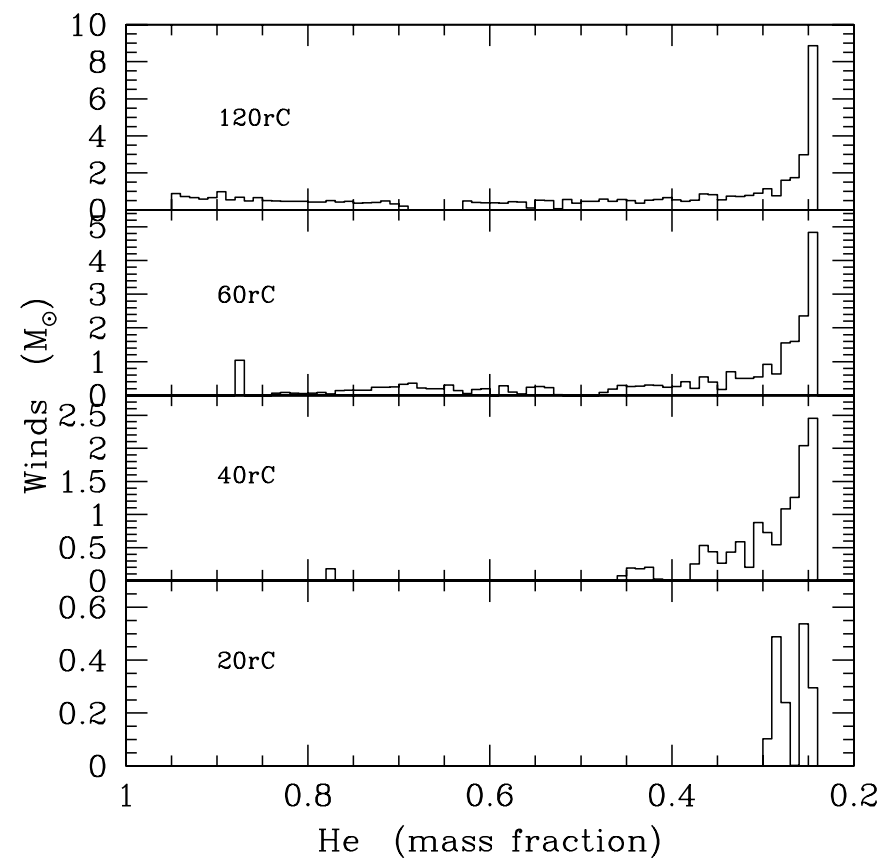

Fig. 9. Mass ejected by slow winds with various helium contents for different initial mass models.

possibility to explain these very O-poor stars has been suggested by D'Antona \& Ventura (2007). These authors explain these stars as resulting from a mixing occurring in the observed star itself along the red giant branch. This mixing would occur only in He-rich stars, because in these stars mixing would not be inhibited by $\mu$-gradients.

If we compare our results with the distribution obtained by Carretta et al. (2007a) (lower panel in Fig. 8) we can recover well stars with $[\mathrm{O} / \mathrm{Na}]$ in the range between -1 and 0.7 although the computed distribution does not recover the exact shape of that observed. We however emphasise that the agreement is quite reasonable in view of the very small number of free parameters. Indeed, given a set of fast rotating massive star models and imposing a given value for the ratio of anomalous to normal stars comparable to that observed in NGC 6752, the computed distribution depends on only one parameter which is $a_{\mathrm{min}}$. Nature is probably more complicated. For example, we considered only one rotational velocity for our massive stars, while real stars probably present a distribution of velocities. Some uncertainties pertain to the nuclear reaction rates (see the effect in Fig. 7), which may affect the distribution of the composition of the slow winds and therefore of the second generation stars. Moreover, as briefly indicated above, the computed distribution depends on the dilution factor, a parameter which is difficult to constrain precisely and which we modelled here in a very simple way. In view of all these caveats, we think that the results obtained are quite encouraging. In the next sections, we provide some predictions which might be used in the future to confirm the scenarios presented in this paper.

\section{Helium content, ${ }^{12} \mathrm{C} /{ }^{13} \mathrm{C}$ and ${ }^{16} \mathrm{O} /{ }^{17} \mathrm{O}$ isotopic ratios}

\subsection{He ejecta from massive polluters}

Figure 9 shows the composition in helium (in mass fraction) of the slow winds ejected by the massive star models of Paper I.

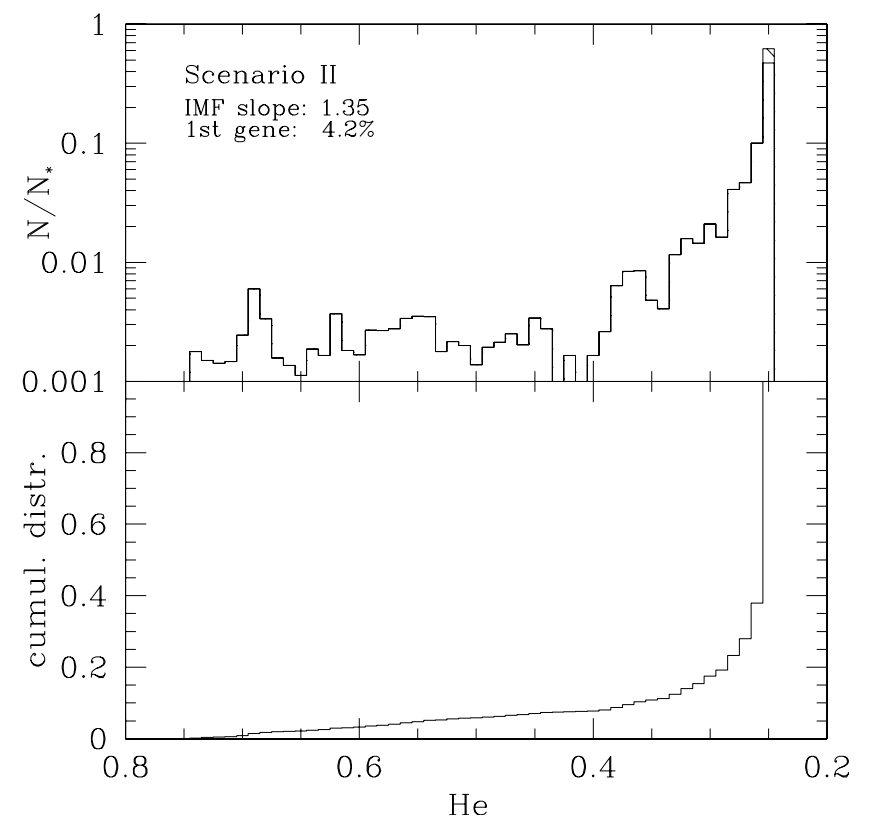

Fig. 10. Top panel: distribution function of He (mass fraction) of lowmass stars for second (white area) and first (hatched area) generation stars. Bottom panel: cumulative distribution function of $\mathrm{He}$ in low-mass stars.

The higher the initial mass of the polluter, the higher the maximum $\mathrm{He}$ value in the wind. For models of 20 and $40 M_{\odot}$ the mass fraction of $\mathrm{He}$ in the wind can reach a value of 0.30 and 0.45 respectively, while it can be as high as 0.80 in more massive models. Such a high value in the latter case is due to the combined action of a more vigorous internal mixing during the evolution of the polluter and especially during the main sequence, and to a stronger mass-loss which reveals H-processed layers. For the $120 M_{\odot}$ the He mass fraction in the wind is already 0.80 at $\mathrm{H}$-exhaustion and reaches 0.90 at the end of the LBV phase.

\subsection{He content of second generation long-lived stars}

Let us now use these predictions for the He content of the slow wind of massive stars, in order to derive the range in the $\mathrm{He}$ content of second generation long-lived stars.

The number fraction of second generation long-lived stars as a function of their helium content is shown in Fig. 10. Only the case for Scenario II is shown (the results would be very similar in the case of Scenario I). The theoretical distribution is that expected at the birth of the second generation stars ${ }^{13}$. An extended tail is present toward very high values (up to 0.75 ) with around $12 \%$ of the low-mass stars displaying a He content higher than 0.4 .

It should be noted that some observational features in globular clusters such as the multiple sequences in $\omega$ Cen (Bedin et al. 2004) and NGC 2808 (Piotto et al. 2007) are explained by an He enrichment of the low-mass stars up to $\mathrm{He} \simeq 0.4$ (Norris 2004; Piotto et al. 2005; D'Antona et al. 2005). Differences in the HB morphology seem also related to high He value (around

\footnotetext{
${ }^{13}$ Internal mixing processes may indeed slightly change the surface composition of stars during their lifetime (for instance during the red giant phase at the first dredge-up the ${ }^{12} \mathrm{C} /{ }^{13} \mathrm{C}$ drops to about 3.5 , with $\mathrm{C}$ and $\mathrm{Li}$ decrease accompanied with an $\mathrm{N}$ increase). Thus the present predictions can only be compared with the observed surface composition of present day low mass stars which are still on the Main Sequence or at an evolutionary stage before the first dredge-up episode.
} 


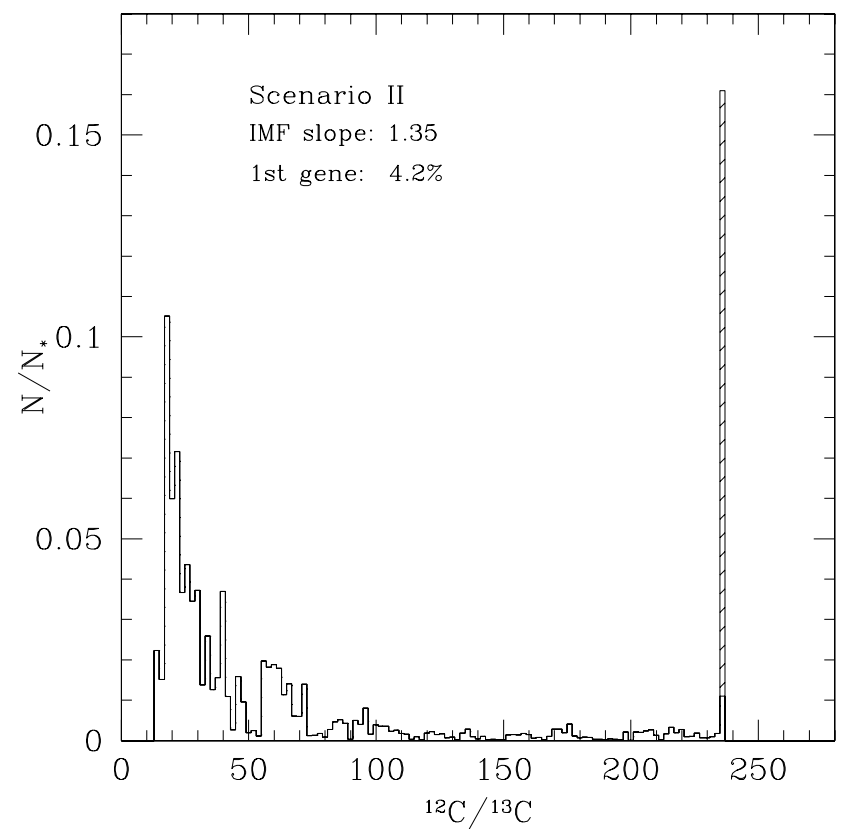

Fig. 11. Number fraction of stars of the second generation composed of material with various ${ }^{12} \mathrm{C} /{ }^{13} \mathrm{C}$ ratios. The hatched area corresponds to first generation stars.

0.4) (D'Antona \& Caloi 2004; D'Antona et al. 2005; Caloi \& D'Antona 2007; Busso et al. 2007). Our model of fast rotating massive stars can easily produce winds with such a value and seem to be a good candidate to be at the origin of these features. However we defer the discussion of this point to a future paper.

\subsection{Carbon isotopic ratio}

We do the same exercise for ${ }^{12} \mathrm{C}$ and ${ }^{13} \mathrm{C}$. Figure 11 shows the number fraction of stars as a function of their ${ }^{12} \mathrm{C} /{ }^{13} \mathrm{C}$ isotopic ratio at birth. Only the case with Salpeter IMF (Scenario II) is shown as the result is almost independent of the adopted IMF. We obtain a bimodal distribution with a broader peak at low ${ }^{12} \mathrm{C} /{ }^{13} \mathrm{C}$ value. The peak around 240 is only made of first generation stars ${ }^{14}$. We note that most of the matter ejected in the slow wind is near $\mathrm{CN}$ equilibrium, i.e., it has ${ }^{12} \mathrm{C} /{ }^{13} \mathrm{C} \sim 3.8$. Dilution with pristine matter shifts the corresponding peak to slightly higher values. Thus many second generation stars are expected to be born with ${ }^{12} \mathrm{C} /{ }^{13} \mathrm{C}$ around $15-20$.

Some observations of the ${ }^{12} \mathrm{C} /{ }^{13} \mathrm{C}$ are available in the literature. For subgiants in NGC 6752, Carretta et al. (2005) found values between 3 and 11 remarkably near the values we obtain for the peak centered on low ${ }^{12} \mathrm{C} /{ }^{13} \mathrm{C}$ values. Thus the ratio in second generation stars is found, as expected, to be low but not at the equilibrium. The observed values are however slightly smaller than the theoretical one. This might indicate a lower dilution factor. Typically, to retrieve the peak around 10, we need to use a dilution factor $a_{\min }$ of 0.1 .

\subsection{Oxygen isotopic ratios}

Let us first recall that, during the core $\mathrm{H}$-burning phase, ${ }^{17} \mathrm{O}$ is produced in stars with initial mass $\leq 40 M_{\odot}$ and is slightly

14 The value of 240 is taken from galactic chemical evolution from Prantzos et al. (1996). A different value around 90 would not change the picture: we would still obtain a bimodal distribution with a high value narrow peak at 90 accompanied with a broad one at lower values.

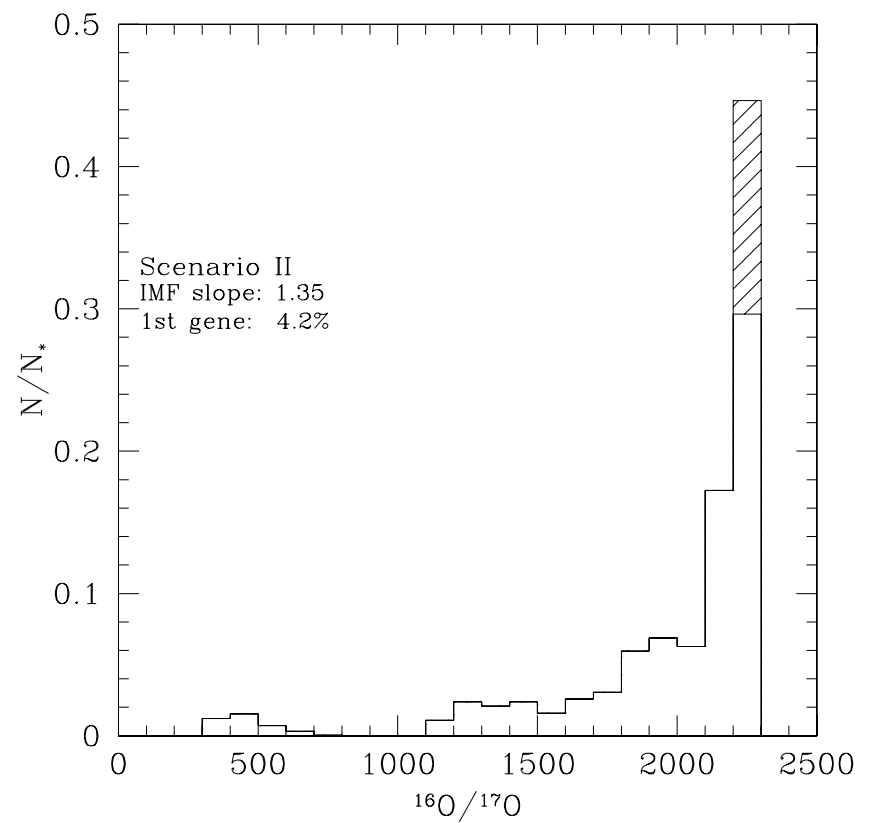

Fig. 12. Number fraction of stars of the second generation composed of material with various ${ }^{16} \mathrm{O} /{ }^{17} \mathrm{O}$ ratios. The hatched area corresponds to first generation stars.

destroyed in more massive stars, while ${ }^{16} \mathrm{O}$ is destroyed in all massive stars. Since in all cases, the burning rate of ${ }^{16} \mathrm{O}$ is faster than that ${ }^{17} \mathrm{O}$, the isotopic ratio ${ }^{15}{ }^{16} \mathrm{O} /{ }^{17} \mathrm{O}$ decreases from 2500 (original value) to values between $300\left(20 M_{\odot}\right)$ and 650 $\left(120 M_{\odot}\right)$.

Figure 12 displays the predictions for the initial composition of anomalous stars obtained for Scenario II with a variable dilution factor $\left(a_{\min }=0.3\right)$. The gap between 400 and 1000 is merely artificial as the lowest values are entirely due to the $20 M_{\odot}$ while the $40 M_{\odot}$ stars start at a ratio of 1000 . So the stars between 20 and $40 M_{\odot}$ will likely fill this gap. We see that only a few percent of stars present ${ }^{16} \mathrm{O} /{ }^{17} \mathrm{O}$ ratios of the order of a few hundred at birth.

\subsection{Expected (anti)correlations}

Figure 13 what are the expected relations between the value of $[\mathrm{O} / \mathrm{Na}]$ in second generation stars at birth and various other chemical characteristics: helium content, $[\mathrm{C} / \mathrm{N}],{ }^{12} \mathrm{C} /{ }^{13} \mathrm{C}$ and ${ }^{16} \mathrm{O} /{ }^{17} \mathrm{O}$ ratios. These (anti)correlations correspond to those which should be observed in globular clusters today provided only non-evolved stars are used, i.e., stars which have not yet undergone any change of their surface abundance due to internal mixing processes. Many interesting points can be seen:

- The relations $\mathrm{He}-[\mathrm{O} / \mathrm{Na}]$ predicted by the different initial mass models present very small dispersions. This indicates that stars observed with a given value of $[\mathrm{O} / \mathrm{Na}]$ should show very similar helium enrichment.

- We see that the dispersion of the $[\mathrm{C} / \mathrm{N}]$ ratio at a given value of $[\mathrm{O} / \mathrm{Na}]$ is quite small if only the most massive stars are considered $\left(M \geq 40 M_{\odot}\right)$. Due to the $20 M_{\odot}$ contribution,

15 The nuclear rates of the reactions ${ }^{17} \mathrm{O}(p, \gamma)$ and ${ }^{17} \mathrm{O}(p, \alpha)$ present large uncertainties for temperatures higher than $100 \times 10^{6} \mathrm{~K}$ which undermine the predictions for explosive nucleosynthesis. However at temperatures reached at the center of main-sequence massive stars (around $50 \times 10^{6} \mathrm{~K}$ ) those uncertainties remain small, at similar level than that of ${ }^{16} \mathrm{O}(p, \gamma)$. 
the dispersion can reach values of $0.4 \operatorname{dex}$ in $[\mathrm{C} / \mathrm{N}]$ at $[\mathrm{O} / \mathrm{Na}]$ equal to about -0.5 . In general, the higher the initial mass, the higher the $[\mathrm{C} / \mathrm{N}]$ ratio for a given $[\mathrm{O} / \mathrm{Na}]$ ratio. This reflects the fact noted above that the slow wind of lower initial mass stars contains mainly advanced processed material (low $[\mathrm{C} / \mathrm{N}]$ values) because they reach the critical velocity only at a late time during the main-sequence phase.

- The dispersion of the ${ }^{12} \mathrm{C} /{ }^{13} \mathrm{C}$ ratio decreases for lower values of $[\mathrm{O} / \mathrm{Na}]$. All the stars with $[\mathrm{O} / \mathrm{Na}]$ ratios below about -0.6 are predicted to show values of the ${ }^{12} \mathrm{C} /{ }^{13} \mathrm{C}$ ratios between 15 and 20.

- The isotopic ratio ${ }^{16} \mathrm{O} /{ }^{17} \mathrm{O}$ presents large dispersions for a given $[\mathrm{O} / \mathrm{Na}]$ ratio on nearly the whole range of $[\mathrm{O} / \mathrm{Na}]$ values. In the case that only very massive stars $\left(M \gtrsim 60 M_{\odot}\right)$ would contribute to provide the material rich in H-burning products, this ratio would remain above 2000 .

It would be of course interesting to study to what extent these relations depend also on the rotational velocities considered, on the initial metallicity and on the mass range. It is of course the hope that such predictions will stimulate new observations which will provide further constraints on the origin of (part of) the matter which was used to form the anomalous stars.

\section{Recall of the main results and discussion}

In this paper we have explored some consequences of the "winds of fast rotating massive stars" scenario described in Paper I.

The two scenarios recalled above involve three free parameters: the slope of the IMF of the polluters, the dilution factors (either the global $d$, or the local $a_{t}$ ), and the number fraction of stars of the first generation which has been lost by the cluster during its lifetime.

To constrain the values of these free parameters we used the following observed features: the observed number ratio of anomalous to normal stars and the Li-Na anticorrelation observed for turnoff stars. Given a set of stellar models, this last feature depends only on the local dilution factor $a_{t}$. From the observed Li-Na anticorrelation in NGC 6752 by Pasquini et al. (2005) and adopting the Eq. (28) for $a_{t}$, we obtain a value of 0.3 for $a_{\mathrm{min}}$, the minimum value of the dilution factor (see Sect. 5.5). As expressed by the form of $a_{t}$, the mixing occurs in the vicinity of the mass losing star. The degree of mixing varies as a function of the mass losing star evolution. Inserting the expression for $a_{t}$ in Eq. (10), we obtain a global dilution factor $d$ of the order of one. It means that the total mass of second generation stars amounts to about twice the total mass released under the form of slow winds by massive stars.

Given the above values for the dilution factors, the observed number ratio of anomalous to normal stars depends only on the two remaining free parameters. In Scenario I, the cluster loses no stars and no stellar remnants. In this case, the IMF is constrained. In Scenario II, the slope of the IMF is set to 1.35 and $e_{\mathrm{LL}}^{1 \mathrm{G}}$ is constrained. To produce a high number of polluted stars (typically 5.7 times the number of normal stars as observed in NGC 6752) we need either (a) in Scenario I, a flat IMF with a slope for massive stars around 0.55 or (b) in Scenario II, that about $96 \%$ of the unpolluted low-mass stars has been lost by the cluster.

Using the two sets of free parameters obtained in Scenarios I and II, we produced predictions for the following features: the variation of the number fraction of (non-evolved) long-lived stars as a function of their surface values of [O/Na], of helium, of ${ }^{12} \mathrm{C} /{ }^{13} \mathrm{C}$ and of ${ }^{16} \mathrm{O} /{ }^{17} \mathrm{O}$, the relations between the $[\mathrm{O} / \mathrm{Na}]$ ratios and the helium, ${ }^{12} \mathrm{C} /{ }^{13} \mathrm{C},{ }^{16} \mathrm{O} /{ }^{17} \mathrm{O}$ and $[\mathrm{C} / \mathrm{N}]$ ratios. Predictions for the mass of the present day globular clusters expressed as fractions of the mass of the gas which has been used to form stars has also been obtained, as well as how the present day mass is distributed among first and second long-lived generation stars, and stellar remnants.

The following results have been obtained:

- The way the number fraction of stars varies as a function of the surface composition is similar in the two scenarios provided the adopted free parameters give the same number ratio of anomalous to normal stars and the same dilution factors.

- In both scenarios, we can produce a $[\mathrm{O} / \mathrm{Na}]$ anticorrelation in the range $-1 \leq[\mathrm{O} / \mathrm{Na}] \leq 0.62$. Quite remarkably, this range corresponds to that observed in NGC 6752 if one considers only the set of stars for which both measurements of oxygen and sodium are available. The possible super-O-poor stars, whose presence is indirectly inferred in some globular clusters, need a lower dilution coefficient or some additional mixing occurring in the star itself.

- In both scenarios with our adopted free parameters, around $12 \%$ of the low-mass stars are expected to display a $\mathrm{He}$ content higher than 0.4 (in mass fraction). Note that Heenrichment is obtained keeping the sum $\mathrm{C}+\mathrm{N}+\mathrm{O}$ equals to its initial value as requested by the observations.

- A tight anti-correlation is predicted between the helium abundance and the value of the ratio $[\mathrm{O} / \mathrm{Na}]$ at the surface of turnoff stars. How this anti-correlation is modified by some internal mixing occurring during the ascent of the red giant branch will be studied in a forthcoming paper.

- More than $30 \%$ of stars are expected to have surface ${ }^{12} \mathrm{C} /{ }^{13} \mathrm{C}$ ratios between 10 and 30 .

- The ratios ${ }^{12} \mathrm{C} /{ }^{13} \mathrm{C}$ and $[\mathrm{O} / \mathrm{Na}]$ are correlated. All the stars with $[\mathrm{O} / \mathrm{Na}]$ ratios below about -0.6 are predicted to show values of the ${ }^{12} \mathrm{C} /{ }^{13} \mathrm{C}$ ratios between 15 and 20 .

- Only a few percent of stars present ${ }^{16} \mathrm{O} /{ }^{17} \mathrm{O}$ ratios of the order of a few hundred at birth.

- A correlation with very a large dispersion is predicted between the ratios ${ }^{16} \mathrm{O} /{ }^{17} \mathrm{O}$ and $[\mathrm{O} / \mathrm{Na}]$. In the case that only stars more massive than about $60 M_{\odot}$ would contribute to the building-up of the chemical anomalies in globular clusters, then only values above 2000 would be expected for this ratio.

- A correlation between $[\mathrm{C} / \mathrm{N}]$ and $[\mathrm{O} / \mathrm{Na}]$ is expected, whose dispersion increases at lower $[\mathrm{O} / \mathrm{Na}]$ values.

- In the case of Scenario I (IMF slope of $0.55, e_{\mathrm{LL}}^{1 \mathrm{G}}=e_{\mathrm{LL}}^{2 \mathrm{G}}=$ $\left.e_{\mathrm{rem}}^{1 \mathrm{G}}=e_{\mathrm{rem}}^{2 \mathrm{G}}=0, a_{\mathrm{min}}=0.3\right)$, the present day mass cluster represents about half of the mass which has been used to form stars. The rest consists of matter ejected by stars and lost by the clusters. The mass locked into remnants represents $19 \%$ of the mass of the present day cluster, the first and second generation stars respectively 12 and $69 \%$.

- In the case of Scenario II (IMF slope of $1.35, e_{\mathrm{LL}}^{\mathrm{iG}}=e_{\mathrm{rem}}^{1 \mathrm{G}}=$ $\left.0.958, a_{\min }=0.3\right)$, the present day mass cluster represents about $9 \%$ of the mass which has been used to form stars. The rest consists of matter ejected by stars and of stars and stellar remnants lost by the cluster. The mass locked into remnants represents $8 \%$ of the mass of the present day cluster, the first and second generation stars respectively 16 and $76 \%$.

Obviously our two scenarios are schematic views of the real formation of globular clusters. We review their respective advantages and drawbacks in Sect. 7.2. 

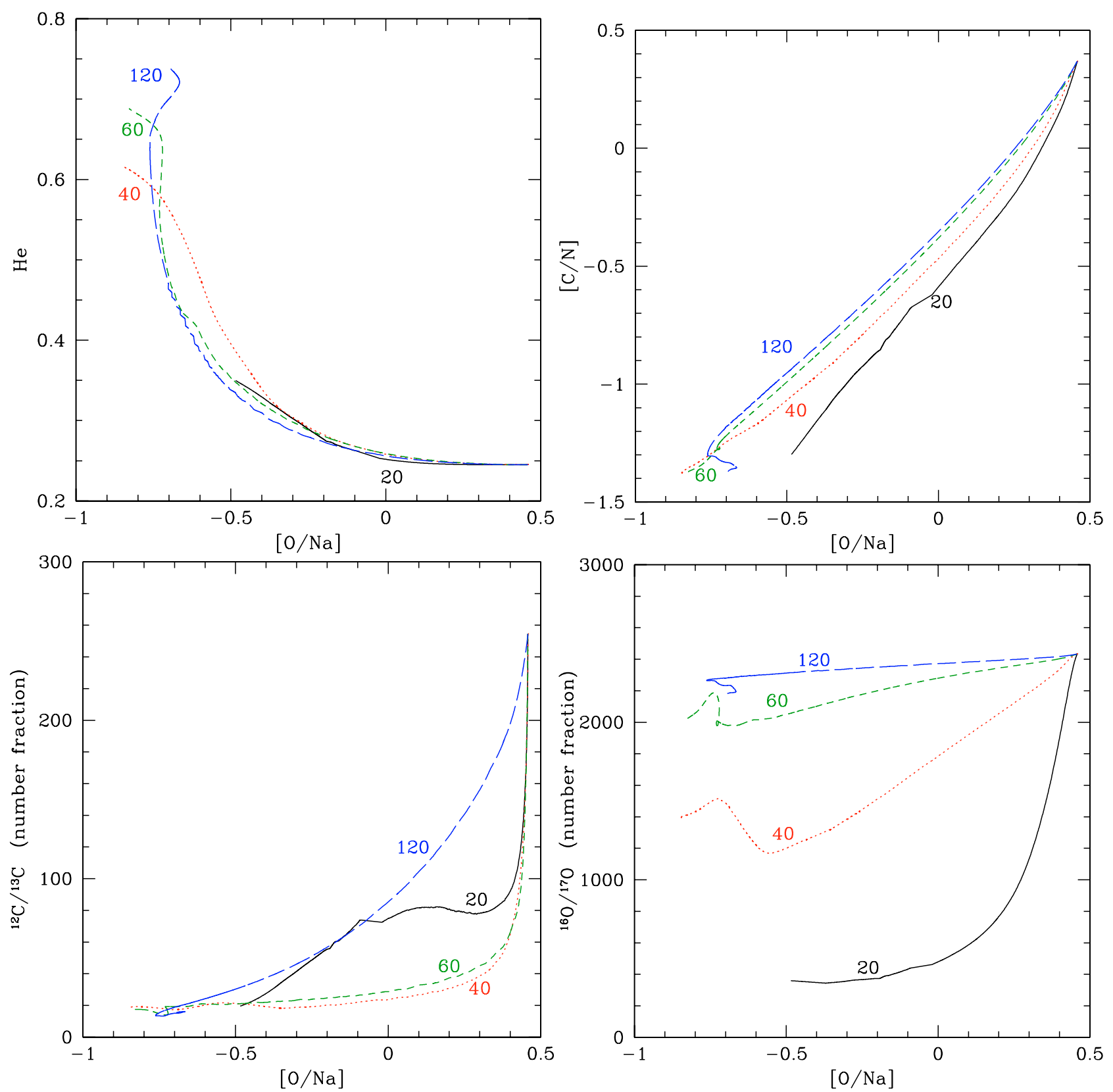

Fig. 13. Upper left: expected anti-correlation between helium and $[\mathrm{O} / \mathrm{Na}]$ for second generation stars (at birth!) born from material ejected through slow winds of different initial mass models. Upper right: same as the upper left panel for the correlation between $[\mathrm{C} / \mathrm{N}]$ and $[\mathrm{O} / \mathrm{Na}]$. Lower left: same as the upper left panel for the correlation between ${ }^{12} \mathrm{C} /{ }^{13} \mathrm{C}$ and $[\mathrm{O} / \mathrm{Na}]$. Lower right: same as the upper left panel for the correlation between ${ }^{16} \mathrm{O} /{ }^{17} \mathrm{O}$ and $[\mathrm{O} / \mathrm{Na}]$.

\subsection{Comparisons with other globular clusters}

In the present study, we have provided some constraints on the slope of the IMF, on the degree of evaporation of the cluster and on the dilution factor based on observations of the globular cluster NGC 6752. We consider to what extent using other globular clusters, the conclusion would have been different.

First, let us recall the fact that the dilution factors have been constrained on the basis of the Li-Na anticorrelation observed at the surface of turnoff stars in NGC 6752. In the absence of similar data for other clusters, we assume that the same dilution factor applies in all globular clusters.

Thus for other globular clusters the other two observed feature which can be used to constrain the slope of the IMF of the polluters and the degree of evaporation of stars are the observed ratio of anomalous to normal stars and the distribution of anomalous stars with given chemical abundances.

The observed ratio of anomalous to normal stars in NGC 2808 (Carretta et al. 2006), NGC 6752 (Carretta et al. 2007a), and NGC 6218 (Carretta et al. 2007b) are respectively $30 \%$ (PC06), 15\% and 15\%. As discussed in Sect. 5.2, a higher proportion of first generation stars induces a steeper IMF in both scenarios (see Fig. 4). In the case of Scenario I, the IMF slope remains much flatter than a Salpeter IMF even when the fraction of first generation stars amounts to $30 \%$. In the framework of Scenario II, a higher proportion of first generation stars allows the cluster to lose less first generation stars, given an IMF slope. However, passing from $15 \%$ to $30 \%$ for the fraction of first generation stars will not change a lot the degree of 


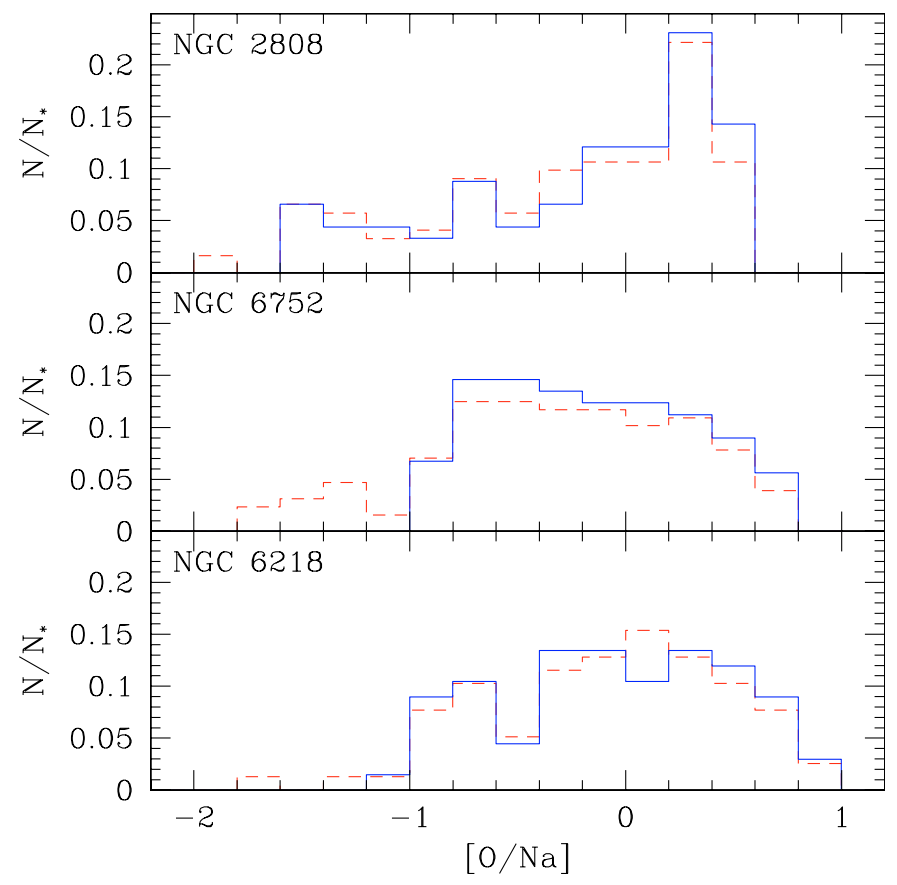

Fig. 14. Observed $[\mathrm{O} / \mathrm{Na}]$ distribution function in NGC 2808 (top), NGC 6752 (middle), and NGC 6218 (bottom). Full lines indicate distribution function obtained with detection or limits of $\mathrm{O}$ in stars while dashed lines indicate distribution obtained by a global anti-correlation relationship to obtain $\mathrm{O}$ abundance.

evaporation. From Fig. 4, we can roughly estimate that it will cause the value of $e_{\mathrm{LL}}^{1 \mathrm{G}}$ decrease from 0.958 to about 0.85 .

Figure 14 displays the observed distribution function of the $[\mathrm{O} / \mathrm{Na}]$ ratio in NGC 2808, NGC 6752, and NGC 6218. Despite that the three globular clusters have similar metallicity, they present distinct shapes for their $[\mathrm{O} / \mathrm{Na}]$ distribution function. In particular, NGC 2808 presents a large population of super-Opoor stars. In NGC 6752 the presence of super-O-poor stars is only inferred from the Na abundance, and NGC 6218 has almost no such stars. These differences are unlikely to be due to an observational bias since the globular clusters have been studied with the same protocol. In fact they could reflect different dynamical histories among the globular clusters. It should be noted that the present day cluster mass of NGC 2808 is higher than that of NGC 6752, and NGC 6752 is heavier than NGC 6218. This is in line with the finding of Carretta (2006) of indications that the GCs with higher masses have more pronounced abundance variations. With a similar IMF a more massive cluster will have more massive stars available to create the abundance anomalies. In addition we can expect that more massive clusters have a deeper potential well and so a better ability to retain the slow winds of massive stars.

From the above considerations, we can thus conclude that using other globular clusters to constrain the free parameters of our two scenarios would not significantly change our conclusions concerning the slope of the IMF or the degree of evaporation of stars. However, probably other parameters, such as the initial mass of the cluster, enter into play in the global picture. We defer the discussion of this point to another paper where further aspects of the dynamical history of the clusters will be accounted for.

\subsection{The pros and cons of the two scenarios}

Finally we compare the two scenarios explored in this paper.

An advantage of Scenario I is that it allows the cluster to retain its first generation of stars and thus to suffer no or negligible evaporation. Looking at the mass of young globular clusters, it seems a reasonable hypothesis (Östlin et al. 2007). Indeed these young globular clusters present masses in the range of the observed masses of present day old galactic globular clusters. Of course we are not sure that the young globular clusters we see today are formed in the same way as those which gave birth to the old galactic globular clusters. Most of the young globular clusters are formed during galaxy merging, while at least part of the old galactic globular clusters may have been formed by a rapid collapse a proto-halo cloud. On the other hand a disadvantage of Scenario I is that it imposes a very flat IMF. At the moment there is no direct empirical evidence that the IMF depends on any physical parameters such as for instance star formation efficiency, the metallicity or the stellar density (Massey et al. 1995). Thus this is probably at the present time a drawback of this scenario.

The advantage of Scenario II is that it allows the use of a standard slope for the IMF. The disadvantage however is that it imposes a very serious loss of first generation stars. We propose a reasonable explanation for why only first generation stars are lost based on the mass segregation of massive stars, but we must admit that the requirement to lose more than $95 \%$ of this generation of stars might be too demanding. Baumgardt \& Kroupa (2007) compute a grid of globular clusters ( $N$-body simulations) to study the effect of residual-gas expulsion on the survival rate and final properties of star clusters. They found that some survival clusters could lose a large part of their initial mass (up to $85 \%$ ). Models of the dynamics of globular clusters with two stellar populations are still required to check whether the strong requirement we need can be achieved or not (e.g., Downing \& Sills 2007).

Thus, we see that whatever scenario is realised, the peculiar abundance patterns observed in globular clusters requires some quite extreme assumptions: either a flat IMF or a very strong evaporation of stars or a combination of the two aspects. Moreover, as explained in more detail in paper I, we have to assume that the first generation of massive stars contained a very high proportion of fast rotators. Is that high proportion of fast rotators due to the peculiar mode of star formation in dense clusters? Are there any other observational hints supporting the existence of these fast rotators? What would be the consequences of this population of massive fast rotators on the frequency of gamma ray bursts in very young globular clusters? We leave open these questions for the moment and we shall probably come back to them in future work. A point however which appears worthwhile to underline here is that massive fast rotators appear to be interesting objects not only to explain the abundance patterns in globular clusters, but also the high N/O ratios observed in very metal poor halo stars (Chiappini et al. 2005, 2006) and some C-rich Ultra Metal Poor Stars (Meynet et al. 2006; Hirschi 2007).

Acknowledgements. The authors wish to thank P. Kroupa and H. Baumgaard for fruitful discussion concerning Scenario II, as well as N. Prantzos. We acknowledge the financial support of the Swiss National Science Foundation (FNS) and of Programme National de Physique Stellaire (PNPS) of CNRS/INSU, France. 


\section{References}

Baumgardt, H., \& Kroupa, P. 2007, ArXiv e-prints, 707

Bedin, L. R., Piotto, G., Anderson, J., et al. 2004, ApJ, 605, L125

Bonifacio, P., Pasquini, L., Molaro, P., et al. 2007, ArXiv e-prints, 704

Busso, G., Cassisi, S., Piotto, G., et al. 2007, ArXiv e-prints, 708

Caloi, V., \& D'Antona, F. 2007, A\&A, 463, 949

Carretta, E. 2006, AJ, 131, 1766

Carretta, E., Gratton, R. G., Lucatello, S., Bragaglia, A., \& Bonifacio, P. 2005, A\&A, 433, 597

Carretta, E., Bragaglia, A., Gratton, R. G., et al. 2006, A\&A, 450, 523

Carretta, E., Bragaglia, A., Gratton, R., Lucatello, S., \& Momany, Y. 2007a, A\&A, 464, 927

Carretta, E., Bragaglia, A., Gratton, R. G., et al. 2007b, ArXiv Astrophysics e-prints

Charbonnel, C. 2005, in IAU Symposium, ed. V. Hill, P. François, \& F. Primas, 347

Charbonnel, C., \& Primas, F. 2005, A\&A, 442, 961

Chiappini, C., Matteucci, F., \& Ballero, S. K. 2005, A\&A, 437, 429

Chiappini, C., Hirschi, R., Meynet, G., et al. 2006, A\&A, 449, L27

D'Antona, F., \& Caloi, V. 2004, ApJ, 611, 871

D’Antona, F., \& Ventura, P. 2007, ArXiv e-prints, 705

D'Antona, F., Bellazzini, M., Caloi, V., et al. 2005, ApJ, 631, 868

Decressin, T., Meynet, G., Charbonnel, C., Prantzos, N., \& Ekström, S. 2007, A\&A, 464, 1029

Denisenkov, P. A., \& Denisenkova, S. N. 1989, Astronomicheskij Tsirkulyar, 1538,11

Denisenkov, P. A., \& Denisenkova, S. N. 1990, Sov. Astron. Lett., 16, 275
Downing, J. M. B., \& Sills, A. 2007, ArXiv Astrophysics e-prints Dufton, P. L., Ryans, R. S. I., Simón-Díaz, S., Trundle, C., \& Lennon, D. J. 2006, A\&A, 451, 603

Gratton, R., Sneden, C., \& Carretta, E. 2004, ARA\&A, 42, 385

Hirschi, R. 2007, A\&A, 461, 571

Huang, W., \& Gies, D. R. 2006, ApJ, 648, 580

Keller, S. C. 2004, Publ. Astron. Soc. Austr., 21, 310

Kroupa, P., \& Boily, C. M. 2002, MNRAS, 336, 1188

Kroupa, P., Tout, C. A., \& Gilmore, G. 1993, MNRAS, 262, 545

Kudryashov, A. D., \& Tutukov, A. V. 1988, Astronomicheskij Tsirkulyar, 1525, 11

Langer, G. E., \& Hoffman, R. D. 1995, PASP, 107, 1177

Langer, G. E., Hoffman, R., \& Sneden, C. 1993, PASP, 105, 301

Massey, P., Johnson, K. E., \& Degioia-Eastwood, K. 1995, ApJ, 454, 151

Meynet, G., Ekström, S., \& Maeder, A. 2006, A\&A, 447, 623

Norris, J. E. 2004, ApJ, 612, L25

Östlin, G., Cumming, R. J., \& Bergvall, N. 2007, A\&A, 461, 471

Paresce, F., \& De Marchi, G. 2000, ApJ, 534, 870

Pasquini, L., Bonifacio, P., Molaro, P., et al. 2005, A\&A, 441, 549

Piotto, G., Villanova, S., Bedin, L. R., et al. 2005, ApJ, 621, 777

Piotto, G., Bedin, L. R., Anderson, J., et al. 2007, ApJ, 661, L53

Porter, J. M., \& Rivinius, T. 2003, PASP, 115, 1153

Prantzos, N., \& Charbonnel, C. 2006, A\&A, 458, 135

Prantzos, N., Aubert, O., \& Audouze, J. 1996, A\&A, 309, 760

Prantzos, N., Charbonnel, C., \& Iliadis, C. 2007, submitted

Salpeter, E. E. 1955, ApJ, 121, 161

Steigman, G. 2006, Int. J. Mod. Phys. E, 15, 1

Strom, S. E., Wolff, S. C., \& Dror, D. H. A. 2005, AJ, 129, 809 